\title{
Reversible periodic orbits in a class of 3D continuous piecewise linear systems of differential equations ${ }^{\text {th }}$
}

\author{
V. Carmona ${ }^{a}$, S. Fernández-García ${ }^{a}$, F. Fernández-Sánchez ${ }^{\mathrm{a}, *}$, \\ E. García-Medina ${ }^{\mathrm{a}}$, A. E. Teruel ${ }^{\mathrm{b}}$ \\ ${ }^{a}$ Departamento de Matemática Aplicada II, Universidad de Sevilla, Escuela Técnica \\ Superior de Ingeniería, Camino de los Descubrimientos s/n, 41092 Sevilla, Spain \\ ${ }^{b}$ Departament de Matemàtiques i Informàtica. Universitat de les Illes Balears. Carretera \\ de Valldemossa km. 7.5. 07122 Palma de Mallorca. Spain.
}

\begin{abstract}
The so-called noose bifurcation is an interesting structure of reversible periodic orbits that was numerically detected by Kent and Elgin in the wellknown Michelson system. In this work we perform an analysis of the periodic behavior of a piecewise version of the Michelson system where this bifurcation also exists. This variant is a one-parameterized three-dimensional piecewise linear continuous system with two zones separated by a plane and it is also a representative of a wide class of reversible divergence-free systems.

In the piecewise system, the noose bifurcation involves reversible periodic orbits that intersect the separation plane at two or four points. This work is focused on those reversible periodic orbits that intersect the separation

\footnotetext{
« This work was partially supported by the Ministerio de Ciencia e Innovación, under the projects MTM2009-07849, MTM2010-20907-C02-01 and MTM2011-22751 and by the Consejería de Educación y Ciencia de la Junta de Andalucía (TIC-0130, P08-FQM-03770). The second author is supported by Ministerio de Educación, grant AP2008-02486.

${ }^{*}$ Corresponding author

Email addresses: vcarmona@us.es (V. Carmona), soledad@us.es

(S. Fernández-García), fefesan@us .es (F. Fernández-Sánchez), egarme@us.es

(E. García-Medina), antonioe.teruel@uib.es (A. E. Teruel)
} 
plane twice (RP2-orbits). It is established that for every $T$ between $2 \pi$ and a critical point there exists a unique value of the parameter for which the system has a RP2-orbit with period T. Moreover, this critical value, that separates periodic orbits with two or four intersection points with the separation plane, corresponds to a RP2-orbit that crosses tangentially the separation plane.

It is also proved that in a bifurcation diagram parameter versus period, the curve of this family of periodic orbits has a unique maximum point, which corresponds to the saddle-node bifurcation of periodic orbits that appears in the noose bifurcation.

Keywords: piecewise linear systems, reversible systems, periodic orbits, saddle-node bifurcation

2000 MSC: 34C23, 34C25, 34C45, 37G15

\section{Introduction}

After equilibria, periodic orbits are the simplest solutions of nonlinear dynamical systems. In spite of that, generically, the proof of their existence is not a trivial problem.

Of course, there are local methods that allow to affirm that there exist periodic orbits in neighborhoods of certain singularities of equilibria (Hopf, Bogdanov-Takens, ..., see [13]) or close to global bifurcations (see, for instance, [19]). In the particular case of piecewise linear systems there are also results in this regard (see $[2,5,8,9,20]$ ). However, it is more difficult to answer some global questions as, for example, to determine the size of those neighborhoods in the phase space or in a parameter space, to know 
where the periodic orbits born/die or to prove the existence of the homoclinic/heteroclinic cycle that organizes the periodic behavior.

An interesting phenomenon related to periodic orbits is the so-called noose bifurcation [10], that appears in the well-known Michelson system $[1,12,18,7,22]$,

$$
\left\{\begin{array}{l}
\dot{x}=y, \\
\dot{y}=z, \\
\dot{z}=d^{2}-y-\frac{1}{2} x^{2},
\end{array}\right.
$$

where the dot stands for the derivative with respect to $t$ and, without loss of generalicity, we can assume that $d \geq 0$. This family of time-reversible systems appears in the study of travelling wave solutions of the one-dimensional Kuramoto-Sivashinsky equation [18]. It also arises in the analysis of the unfolding of the nilpotent singularity of codimension three $[6,7]$.

The noose bifurcation occurs when the curve of the family of periodic orbits that appears from a period-doubling bifurcation and the curve of the original family of periodic orbits come together and annihilate at a saddlenode bifurcation, see Fig. 1. Therefore, two of the most common bifurcations of periodic orbits (saddle-node and period-doubling) are connected by a noose-shaped curve.

In [10] the authors perform an excellent study of the noose bifurcation. They associate its existence to the appearance of a small extra loop at the periodic orbit. This loop grows until it coincides with the other loop at the period-doubling bifurcation. The existence of the noose bifurcation is based on numerical computations and no theoretical proofs are given. In fact, although there are many interesting works about periodic behavior and 

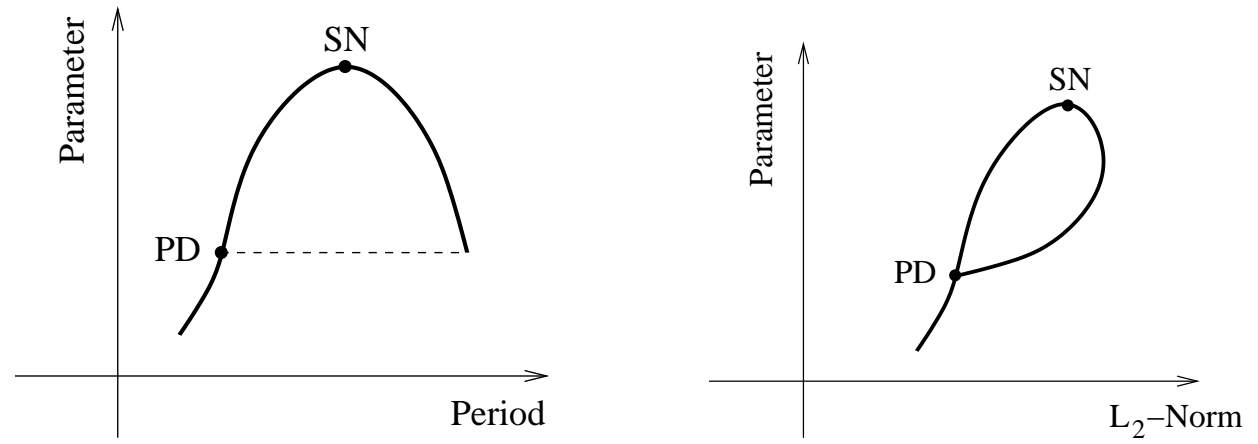

Figure 1: Schematic picture of the noose structure in two different bifurcation diagrams.

global connections in the Michelson system (see, for instance, $[1,6,11,14$, $17,21,22,23])$, as far as we know, the existence of the noose bifurcation has not been proved yet.

On the other hand, Arneodo, Coullet and Tresser in [2] realized that piecewise linear systems gave a good chance of proving analytically the existence of dynamical objects. Following this idea, in [3] and [4] the authors studied some global connections of system

$$
\left\{\begin{array}{l}
\dot{x}=y, \\
\dot{y}=z, \\
\dot{z}=1-y-\lambda\left(1+\lambda^{2}\right)|x|,
\end{array}\right.
$$

where the parameter $\lambda$ is strictly positive. Particularly, they give an analytical proof of the existence of a pair of homoclinic connections and a T-point heteroclinc cycle.

The continuous system (2) is given by two linear systems separated by the plane $\{x=0\}$, called the separation plane, and it can be considered as a continuous piecewise linear version of the Michelson system (1). In fact, a simple linear change of variables followed by the change of function 
$x^{2} \rightarrow|x|$ allows to obtain system (2) from system (1), see [3]. Moreover, both systems are volume-preserving and time-reversible with respect to the involution $\mathbf{R}(x, y, z)=(-x, y,-z)$. Some other dynamical aspects of the Michelson system also remain in this piecewise linear version [3, 4].

Specifically, we have checked numerically that the system (2) also exhibits the complete structure of reversible periodic orbits related to the noose bifurcation (see Fig.2). In this figure, together with the saddle-node (SN) and period doubling $(\mathrm{PD})$ bifurcations we would like to focus the attention on the existence of a point (TG) where a qualitative change occurs to the shape of the periodic orbit. Concretely, the solid line corresponds to reversible periodic orbits that intersect exactly twice the separation plane while the dashed one stands for reversible periodic orbits with exactly four intersections with the separation plane. The transition between these two kinds of periodic orbits involves the existence of a periodic orbit that has a transversal tangency with the separation plane. This tangency creates the small loop that finally closes the noose.

Although this numerical analysis may convince us of the existence of the noose bifurcation in system (2), we have decided that we could go farther if we were able to give a proof by hand of the existence of this complete structure. From our point of view, this proof should have to be divided at least in two different parts, depending on the number of intersections between the periodic orbits and the separation plane. This is even more evident if we take into account the closing equations technique that we are going to use below.

This work concerns only with reversible periodic orbits which intersect 


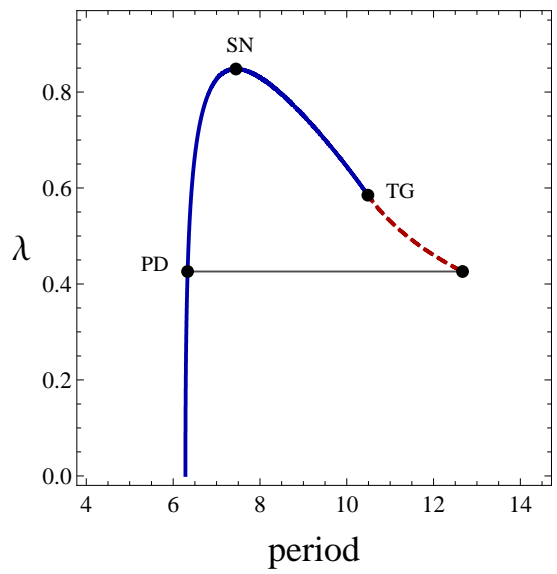

Figure 2: Noose bifurcation in the piecewise linear continuous system (2). The saddlenode (SN), period-doubling (PD) and tangency (TG) points are also shown. The thick solid/dashed line corresponds to reversible periodic orbits which have exactly two/four intersections with the separation plane. A thin line joins the periodic orbits involved in the period-doubling bifurcation.

exactly twice the separation plane. These orbits will be denoted by RP2 (Reversible Periodic orbit with 2 intersections). Concretely, a global result for their existence is established.

The system (2) can be written in matrix form as

$$
\dot{\mathbf{x}}=\left\{\begin{array}{cc}
A_{+} \mathbf{x}+\mathbf{e}_{3} & \text { if } x \geq 0, \\
A_{-} \mathbf{x}+\mathbf{e}_{3} & \text { if } x \leq 0
\end{array}\right.
$$

with

$$
A_{ \pm}=\left(\begin{array}{ccc}
0 & 1 & 0 \\
0 & 0 & 1 \\
\mp \lambda\left(1+\lambda^{2}\right) & -1 & 0
\end{array}\right), \mathbf{x}=\left(\begin{array}{l}
x \\
y \\
z
\end{array}\right) \text { and } \mathbf{e}_{3}=\left(\begin{array}{c}
0 \\
0 \\
1
\end{array}\right) \text {. }
$$

In the half-space $\{x<0\}$, the system has exactly one saddle-focus equilib- 
rium point $\mathbf{p}_{-}=\left(-1 /\left(\lambda\left(1+\lambda^{2}\right)\right), 0,0\right)^{T}$, since the eigenvalues of matrix $A_{-}$ are $\lambda$ and $\alpha \pm \mathrm{i} \beta$, where

$$
\alpha=-\frac{\lambda}{2} \text { and } \beta=\frac{\sqrt{4+3 \lambda^{2}}}{2} .
$$

By the reversibility with respect to $\mathbf{R}$, there exists exactly one saddle-focus equilibrium $\mathbf{p}_{+}=-\mathbf{p}_{-}$in the half-space $\{x>0\}$ and its eigenvalues are given by $-\lambda$ and $-\alpha \pm \mathrm{i} \beta$.

Taking into account that both linear systems correspond to saddle-focus equilibria, it is obvious that a periodic orbit must live in both half-spaces, $\{x>0\}$ and $\{x<0\}$. Hence, the periodic orbits of system (2) have to intersect the separation plane $\{x=0\}$ at least at two points.

Before developing the conditions for the existence of RP2-orbits it is convenient to describe some basic elements of the flow of system (2). For instance, the stable two-dimensional manifold $W^{s}\left(\mathbf{p}_{-}\right)$of equilibrium point $\mathbf{p}_{-}$ is locally contained in the half-plane

$$
\mathcal{P}_{-}=\left\{\lambda\left(1+\lambda^{2}\right) x+\lambda^{2} y+\lambda z=-1, x \leq 0\right\},
$$

which is called the focal half-plane of $\mathbf{p}_{-}$. This half-plane is obtained from the eigenvectors associated to the complex eigenvalues of $A_{-}$. The halfplane $\mathcal{P}_{-}$and the separation plane $\{x=0\}$ intersect along the straight-line $\mathcal{D}_{-}=\left\{x=0, \lambda^{2} y+\lambda z=-1\right\}$.

The unstable manifold $W^{u}\left(\mathbf{p}_{-}\right)$of $\mathbf{p}_{-}$contains the half-line $\mathcal{L}_{-}=\left\{\mathbf{p}_{-}-\right.$ $\left.\mu\left(1, \lambda, \lambda^{2}\right):-\frac{1}{\lambda\left(1+\lambda^{2}\right)} \leq \mu<\infty\right\}$ generated by the eigenvector associated to the eigenvalue $\lambda$ of the matrix $A^{-}$.

The elements in the other half-space can be obtained by using the involution $\mathbf{R}$. So, the stable manifold $W^{s}\left(\mathbf{p}_{+}\right)$of $\mathbf{p}_{+}$contains the half-line 
$\mathcal{L}_{+}=\left\{\mathbf{p}_{+}+\mu\left(1,-\lambda, \lambda^{2}\right):-\frac{1}{\lambda\left(1+\lambda^{2}\right)} \leq \mu<\infty\right\}$ and the unstable twodimensional manifold $W^{u}\left(\mathbf{p}_{+}\right)$is locally contained in the half-plane

$$
\mathcal{P}_{+}=\left\{\lambda\left(1+\lambda^{2}\right) x-\lambda^{2} y+\lambda z=1, x \geq 0\right\},
$$

which is called the focal half-plane of $\mathbf{p}_{+}$. The half-plane $\mathcal{P}_{+}$and the separation plane $\{x=0\}$ intersect along the straight-line $\mathcal{D}_{+}=\left\{x=0,-\lambda^{2} y+\lambda z=1\right\}$.

The intersection between the straight-lines $\mathcal{D}_{+}$and $\mathcal{D}_{-}$is given by the point $\mathbf{q}=\left(0,-1 / \lambda^{2}, 0\right)$.

Taking into consideration that system (2) is formed by two linear systems separated by the plane $\{x=0\}$, it is also interesting to understand the behaviour of the flow crossing this plane. From the first equation of the system, it is clear that an orbit which intersects the plane $\{x=0\}$ at a point $\left(0, y_{0}, z_{0}\right)$ with $y_{0}>0$, crosses transversally the separation plane from $\{x<0\}$ to $\{x>0\}$. Analogously, when $y_{0}<0$ the orbit will cross transversally the separation plane from $\{x>0\}$ to $\{x<0\}$. In the case $y_{0}=0$, the local shape of the orbit depends on the sign of $z_{0}$ : for $z_{0}>0$ the orbit is locally contained in $\{x \geq 0\}$, for $z_{0}<0$ the orbit is locally contained in $\{x \leq 0\}$ and for $z_{0}=0$ the orbit crosses tangentially the plane $x=0$ from $\{x<0\}$ to $\{x>0\}$. The $z$-axis is so-called the tangency line. More details can be found in $[4,16]$.

Coming back to periodic orbits, we are going to obtain now a set of conditions to characterize RP2-orbits. It is well known that a periodic orbit is reversible if and only if it intersects twice the set of fixed points of involution $\mathbf{R}$, which in this case corresponds to the $y$-axis. Let $\mathbf{x}\left(t ; \lambda, y_{0}\right)=$ $\left(x\left(t ; \lambda, y_{0}\right), y\left(t ; \lambda, y_{0}\right), z\left(t ; \lambda, y_{0}\right)\right)$ stands for the solution of system (2) with initial condition $\mathbf{x}\left(0 ; \lambda, y_{0}\right)=\left(0, y_{0}, 0\right)$. Assume that there exist three real 
values $\hat{t}>0, \hat{\lambda}>0$ and $\hat{y}_{0}$ such that

$$
\begin{aligned}
& \mathbf{x}\left(\hat{t} ; \hat{\lambda}, \hat{y}_{0}\right) \in \operatorname{Fix}(\mathbf{R}), \\
& \hat{y}_{0}<0 \\
& x\left(t ; \hat{\lambda}, \hat{y}_{0}\right)<0 \text { for all } t \in(0, \hat{t}) .
\end{aligned}
$$

Let us call $\hat{y}_{1}=y\left(\hat{t} ; \hat{\lambda}, \hat{y}_{0}\right), \hat{\mathbf{p}}_{0}=\left(0, \hat{y}_{0}, 0\right)$ and $\hat{\mathbf{p}}_{1}=\left(0, \hat{y}_{1}, 0\right)$. Then, under hypotheses (5)-(7), system (2) has for $\lambda=\hat{\lambda}$ a RP2-orbit whose period is $2 \hat{t}$ (half-period is $\hat{t}$ ) and whose intersections with the separation plane are $\hat{\mathbf{p}}_{0}$ and $\hat{\mathbf{p}}_{1}$. Note that when $\hat{y}_{1}=0$ the periodic orbit crosses tangentially the plane $\{x=0\}$ at $\hat{\mathbf{p}}_{1}=(0,0,0)$.

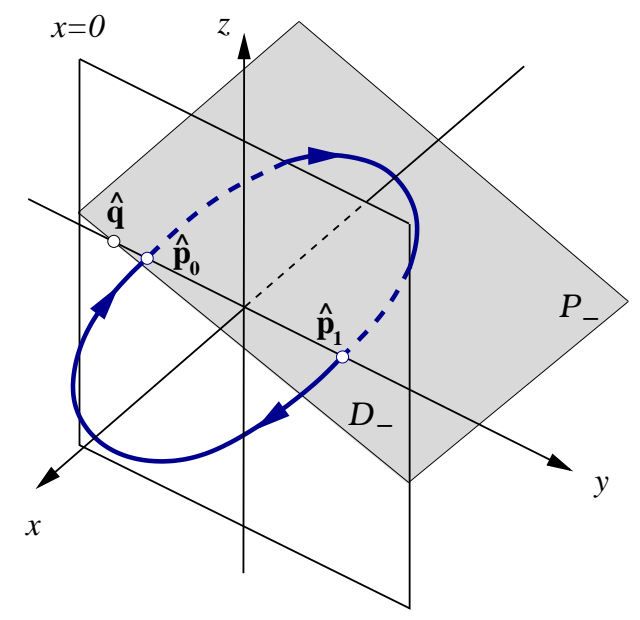

Figure 3: Reversible periodic orbit of system (2) which has exactly two transversal intersections with $\{x=0\}$.

In Fig. 3, a generic RP2-orbit is shown. The focal half-plane $\mathcal{P}_{-}$and the points $\hat{\mathbf{p}}_{0}, \hat{\mathbf{p}}_{1}$ and $\hat{\mathbf{q}}=\left(0,-1 / \hat{\lambda}^{2}, 0\right)$, which are contained in $\operatorname{Fix}(\mathbf{R})$, are also 
represented. Note that

$$
\hat{y}_{0}>-\frac{1}{\hat{\lambda}^{2}}
$$

because, obviously, the RP2-orbit cannot intersect the focal half-planes. Moreover, from hypotheses (5)-(7) and the properties of the flow through the separation plane, the inequality

$$
y_{1}=y\left(\hat{t} ; \hat{\lambda}, \hat{y}_{0}\right) \geq 0
$$

must be fulfilled.

Condition (5) leads to a system of two equations and three unknowns, $\left(t, \lambda, y_{0}\right)$. Section 2 is devoted to explore its solution set. Concretely, we will replace $t$ with $\tau=\sqrt{4+3 \lambda^{2}} t / 2$ and thus we will prove that non-trivial solutions are parameterized by $\tau \in \cup_{n \geq 1}[(2 n-1) \pi, 2 n \pi]$. In Section 3 the analysis will be restricted to the first interval, $\tau \in[\pi, 2 \pi]$, and we will study the points verifying condition (5) that also correspond to RP2-orbits, that is, those which satisfy conditions (6)-(7). In Section 4, two results will be proved. On the one hand, the unique RP2-orbits of system (2) whose period is less than $4 \pi$ are those obtained in Section 3 and, on the other hand, there exists a fold bifurcation of periodic orbits. Finally, in Section 5 we dedicate some lines to RP2-orbits with period greater than $4 \pi$.

The following theorem, which is the core of the paper, is obtained from the analysis performed in Sections 2-4.

Theorem 1. There exist two values $0<\lambda_{C}<\lambda_{F}$ such that the following statements hold:

1. If $\lambda \in\left(0, \lambda_{C}\right)$ system (2) has a unique RP2-orbit whose period is less than $4 \pi$. 


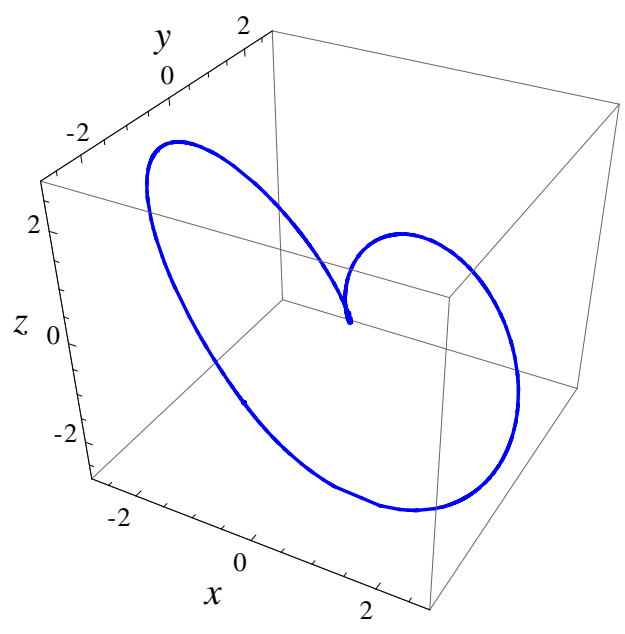

Figure 4: Reversible periodic orbit which crosses tangentially the separation plane $\{x=0\}$.

2. For $\lambda=\lambda_{C}$ system (2) has exactly two RP2-orbits with periods less than $4 \pi$. Moreover, those periods are different and the corresponding RPQ-orbit of longer period crosses tangentially the plane $\{x=0\}$.

3. If $\lambda \in\left(\lambda_{C}, \lambda_{F}\right)$ system (2) has exactly two RP2-orbits with periods less than $4 \pi$. Moreover, those periods are different.

4. For $\lambda=\lambda_{F}$ system (2) has a unique RP2-orbit whose period is less than $4 \pi$.

5. If $\lambda>\lambda_{F}$ system (2) does not have any RP2-orbits with period less than $4 \pi$.

The periodic orbit that crosses tangentially the plane $\{x=0\}$ for $\lambda=\lambda_{C}$ is shown in Fig. 4. The bifurcation diagram ( $\lambda$ vs. half-period) described in Theorem 1 for RP2-orbits is shown in Fig. 5. Numerical computations allow to obtain $\lambda_{C} \approx 0.5851$ and $\lambda_{F} \approx 0.8481$.

Note that we have chosen the subscript $F$ for the value of $\lambda$ given in 


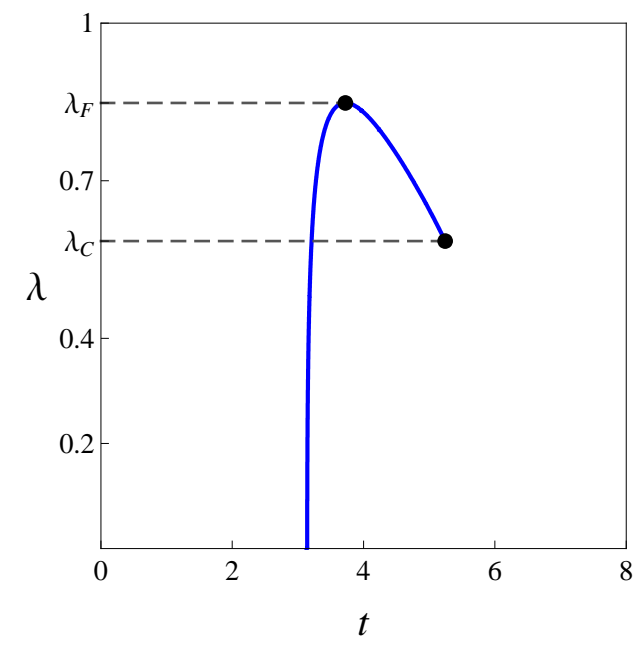

Figure 5: Curve of RP2-orbits in plane $t$ - $\lambda$, where $t$ is the half-period and the values $\lambda_{C}$ and $\lambda_{F}$ are given in Theorem 1.

statement 4 of Theorem 1 because this value corresponds to a fold or saddlenode bifurcation of periodic orbits. This fact is also proved below.

\section{Analysis of the closing equations}

This section is devoted to investigate the set of points which satisfy condition (5), that can be easily written as the following system of two equations

$$
\left\{\begin{array}{l}
x\left(\hat{t} ; \hat{\lambda}, \hat{y}_{0}\right)=0 \\
z\left(\hat{t} ; \hat{\lambda}, \hat{y}_{0}\right)=0 .
\end{array}\right.
$$

According to conditions (6) and (7), the expressions of $x$ and $z$ in system (10) can be obtained by integrating the linear system corresponding to the half-space $\{x<0\}$. Thus, after suitable manipulations, it is obvious that $\left(\hat{t}, \hat{\lambda}, \hat{y}_{0}\right)$ verifies system $(10)$ if and only if $\left(t, \lambda, y_{0}\right)=\left(\hat{t}, \hat{\lambda}, \hat{y}_{0}\right)$ is a solution 
of system

$$
\left\{\begin{array}{l}
E_{1}\left(t, \lambda, y_{0}\right)=0 \\
E_{2}\left(t, \lambda, y_{0}\right)=0
\end{array}\right.
$$

where

$$
\begin{aligned}
E_{1}\left(t, \lambda, y_{0}\right) & =\sqrt{4+3 \lambda^{2}}\left[-1-3 \lambda^{2}+\left(1+\lambda^{2}\right)\left(1+\lambda^{2} y_{0}\right) e^{t \lambda}\right] e^{\frac{t \lambda}{2}} \\
& +\lambda\left[\left(1+\lambda^{2}\right)\left(2+3 \lambda^{2}\right) y_{0}-2\right] \sin (\beta t) \\
& -\lambda^{2} \sqrt{4+3 \lambda^{2}}\left[\left(1+\lambda^{2}\right) y_{0}-2\right] \cos (\beta t),
\end{aligned}
$$

and

$$
\begin{aligned}
E_{2}\left(t, \lambda, y_{0}\right) & =\lambda\left(1+\lambda^{2} y_{0}\right) \sqrt{4+3 \lambda^{2}}\left(e^{\frac{3 \lambda}{2} t}-\cos (\beta t)\right) \\
& +\left[2+3 \lambda^{2}-\left(2+6 \lambda^{2}+3 \lambda^{4}\right) y_{0}\right] \sin (\beta t),
\end{aligned}
$$

with $\beta$ given in equation (4).

Note that system (11) can be read as a system of two first-order linear equations for $y_{0}$,

$$
\left\{\begin{array}{l}
a_{1}(t, \lambda) y_{0}+b_{1}(t, \lambda)=0 \\
a_{2}(t, \lambda) y_{0}+b_{2}(t, \lambda)=0 .
\end{array}\right.
$$

The determinant of the augmented matrix is $\left(1+3 \lambda^{2}\right) \sqrt{4+3 \lambda^{2}} E(t, \lambda)$, where

$$
\begin{aligned}
E(t, \lambda) & =\lambda^{3} \sqrt{4+3 \lambda^{2}}\left[\left(1+e^{2 t \lambda}\right)-\left(1+e^{t \lambda}\right) e^{\frac{t \lambda}{2}} \cos (\beta t)\right] \\
& +\left(2+6 \lambda^{2}+3 \lambda^{4}\right)\left(e^{t \lambda}-1\right) e^{\frac{t \lambda}{2}} \sin (\beta t)
\end{aligned}
$$

So, system (11) can be solved for $y_{0}$ if, and only if,

$$
E(t, \lambda)=0
$$

Moreover, this solution is unique because, as it is proved in the following lemma, the coefficient

$$
a_{2}(t, \lambda)=\left(2+6 \lambda^{2}+3 \lambda^{4}\right) \sin (\beta t)-\lambda^{3} \sqrt{4+3 \lambda^{2}}\left(e^{\frac{3 \lambda}{2} t}-\cos (\beta t)\right)
$$


and the function $E(t, \lambda)$ cannot vanish simultaneously. Therefore, from the second equation of system (11) it is possible to obtain $y_{0}$ as a function of $(t, \lambda)$

$$
y_{0}(t, \lambda)=\frac{\left(2+3 \lambda^{2}\right) \sin (\beta t)+\lambda \sqrt{4+3 \lambda^{2}}\left(e^{\frac{3 \lambda}{2} t}-\cos (\beta t)\right)}{\left(2+6 \lambda^{2}+3 \lambda^{4}\right) \sin (\beta t)-\lambda^{3} \sqrt{4+3 \lambda^{2}}\left(e^{\frac{3 \lambda}{2} t}-\cos (\beta t)\right)} .
$$

Lemma 1. For $t>0$ and $\lambda>0$, system (11) is equivalent to the system formed by equations (12) and (13).

Proof. The following expressions for the trigonometric functions $\sin (\beta t)$ and $\cos (\beta t)$ can be obtained from the system $a_{2}(t, \lambda)=0, E(t, \lambda)=0$.

$$
\sin (\beta t)=\frac{\lambda^{3} \sqrt{4+3 \lambda^{2}}\left(e^{3 t \lambda}-1\right) e^{-\frac{3 t \lambda}{2}}}{2\left(2+6 \lambda^{2}+3 \lambda^{4}\right)}, \quad \cos (\beta t)=\frac{\left(1+e^{3 t \lambda}\right) e^{-\frac{3 t \lambda}{2}}}{2} .
$$

Since

$$
\sin ^{2}(\beta t)+\cos ^{2}(\beta t)-1=\frac{\left(1+\lambda^{2}\right)^{3}\left(1+3 \lambda^{2}\right)\left(e^{3 t \lambda}-1\right)^{2} e^{-3 t \lambda}}{\left(2+6 \lambda^{2}+3 \lambda^{4}\right)^{2}}>0,
$$

for $t>0$ and $\lambda>0$, functions $a_{2}(t, \lambda)$ and $E(t, \lambda)$ cannot vanish simultaneously and the proof is concluded.

As it has been outlined above, the solution set of system (11) can be studied from equation (12). Although $\lambda$ must be positive for our model, the solution set of (12) has several symmetry properties which makes interesting to analyze this set even for non-positive values of $\lambda$.

Note that $E(t, 0)=0$ for every $t$. To remove this limit case we introduce the smooth function

$$
F(t, \lambda)= \begin{cases}\frac{E(t, \lambda)}{\lambda} & \text { if } \quad \lambda \neq 0 \\ 2 t \sin (t) & \text { if } \quad \lambda=0\end{cases}
$$


where the values $F(t, 0)$ have been obtained by continuity. Furthermore, more suitable coordinates are chosen by replacing $t$ with the new variable

$$
\tau=\frac{\sqrt{4+3 \lambda^{2}}}{2} t
$$

So, from now on, we consider the function

$$
G(\tau, \lambda)=F\left(\frac{2 \tau}{\sqrt{4+3 \lambda^{2}}}, \lambda\right)
$$

Some basic properties which describe the zero set of function $G$ are stated in the next lemma.

Lemma 2. The following statements about function $G$ hold.

1. Function $G$ vanishes for $(\tau, \lambda)=(k \pi, 0)$ with $k \in \mathbb{N}^{*}:=\mathbb{N} \cup\{0\}$. Moreover, if $\tau \in(k \pi,(k+1) \pi), k \in \mathbb{N}^{*}$, then $\operatorname{sign}(G(\tau, 0))=(-1)^{k}$.

2. The points $(k \pi, 0)$ with $k \in \mathbb{N}^{*}$ are fold points of the zero set of $G$, that $i s, G(k \pi, 0)=\frac{\partial G}{\partial \lambda}(k \pi, 0)=0$ and $\frac{\partial G}{\partial \tau}(k \pi, 0) \cdot \frac{\partial^{2} G}{\partial \lambda^{2}}(k \pi, 0) \neq 0$.

3. Function $G$ satisfies $G(\tau, \lambda)=\exp \left(\frac{4 \tau \lambda}{\sqrt{4+3 \lambda^{2}}}\right) G(\tau,-\lambda)$ for all $(\tau, \lambda) \in$ $\mathbb{R}^{2}$.

4. If $\tau \in \bigcup_{k \in \mathbb{N}^{*}}(2 k \pi,(2 k+1) \pi)$ then $G(\tau, \lambda)>0$ for all $\lambda \in \mathbb{R}$.

5. For every $\tau \in \bigcup_{k \in \mathbb{N}}[(2 k-1) \pi, 2 k \pi]$ there exists a unique value $\tilde{\lambda}(\tau) \geq 0$ such that $G(\tau, \tilde{\lambda}(\tau))=G(\tau,-\tilde{\lambda}(\tau))=0$. Moreover, if $\tau \in \bigcup_{k \in \mathbb{N}}((2 k-$ 1) $\pi, 2 k \pi)$ then $\tilde{\lambda}(\tau)>0, \frac{\partial G}{\partial \lambda}(\tau, \tilde{\lambda}(\tau))>0$ and $\frac{\partial G}{\partial \lambda}(\tau,-\tilde{\lambda}(\tau))<0$.

6. If $\tau \in \bigcup_{k \in \mathbb{N}}[(2 k-1) \pi, 2 k \pi]$ and $\lambda>0$, then $\operatorname{sign}(G(\tau, \lambda))=\operatorname{sign}(\lambda-$ $\tilde{\lambda}(\tau))$.

7. For every $k \in \mathbb{N}$ there exists a unique pair $\left(\tau_{k}, \lambda_{k}\right) \in((2 k-1) \pi, 2 k \pi) \times$ $(0,+\infty)$ such that $G\left(\tau_{k}, \lambda_{k}\right)=G\left(\tau_{k},-\lambda_{k}\right)=0$ and $\frac{\partial G}{\partial \tau}\left(\tau_{k}, \lambda_{k}\right)=\frac{\partial G}{\partial \tau}\left(\tau_{k},-\lambda_{k}\right)=$ 
0. Moreover, $\frac{\partial^{2} G}{\partial \tau^{2}}\left(\tau_{k}, \lambda_{k}\right) \cdot \frac{\partial^{2} G}{\partial \tau^{2}}\left(\tau_{k},-\lambda_{k}\right) \neq 0$ and so the pairs $\left(\tau_{k}, \lambda_{k}\right)$ and $\left(\tau_{k},-\lambda_{k}\right)$ are fold points of the zero set of $G$.

Proof. The proofs of items 1 and 2 are immediate and the proof of item 3 is a direct consequence of the obvious property $E(t, \lambda)=-e^{2 t \lambda} E(t,-\lambda)$.

The proofs of some of the remaining items are based on geometric reasonings. The principal idea is to write the equation $G(\tau, \lambda)=0$ as a system whose equations correspond to a straight line and the unit circle. More precisely, for $X=\cos (\tau)$ and $Y=\sin (\tau)$ the equation $G(\tau, \lambda)=0$ can be thought of as the system

$$
\left\{\begin{aligned}
r \equiv A(\tau, \lambda)+B(\tau, \lambda) X+C(\tau, \lambda) Y & =0 \\
X^{2}+Y^{2} & =1
\end{aligned}\right.
$$

where, for $\lambda \neq 0$, the coefficients are given by

$$
\begin{aligned}
& A(\tau, \lambda)=\lambda^{2} \sqrt{4+3 \lambda^{2}}\left(1+e^{\frac{4 \tau \lambda}{\sqrt{4+3 \lambda^{2}}}}\right), \\
& B(\tau, \lambda)=-\lambda^{2} \sqrt{4+3 \lambda^{2}}\left(e^{\frac{3 \tau \lambda}{\sqrt{4+3 \lambda^{2}}}}+e^{\frac{\tau \lambda}{\sqrt{4+3 \lambda^{2}}}}\right), \\
& C(\tau, \lambda)=\left(2+6 \lambda^{2}+3 \lambda^{4}\right)\left(\frac{e^{\frac{3 \tau \lambda}{\sqrt{4+3 \lambda^{2}}}}-e^{\frac{\tau \lambda}{\sqrt{4+3 \lambda^{2}}}}}{\lambda}\right)
\end{aligned}
$$

and, for $\lambda=0$, it holds $A(\tau, 0)=B(\tau, 0)=0$ and $C(\tau, 0)=2 \tau$.

It follows that

$$
A(\tau, \lambda) \geq 0, \quad B(\tau, \lambda) \leq 0 \quad \text { and } \quad \operatorname{sign}(C(\tau, \lambda))=\operatorname{sign}(\tau) .
$$

Therefore, a point $(\cos (\tau), \sin (\tau))$, with $\tau>0$, is located above the straight line $r$ if and only if $G(\tau, \lambda)>0$ and it is located below $r$ if and only if $G(\tau, \lambda)<0$. 
The first coordinate of the intersection point between the straight line $r$ and $Y=0$ is

$$
\tilde{X}(\tau, \lambda)=\frac{-A(\tau, \lambda)}{B(\tau, \lambda)}=\frac{1+e^{\frac{4 \tau \lambda}{\sqrt{4+3 \lambda^{2}}}}}{e^{\frac{3 \tau \lambda}{\sqrt{4+3 \lambda^{2}}}}+e^{\frac{\tau \lambda}{\sqrt{4+3 \lambda^{2}}}}} .
$$

Note that function $\tilde{X}$ satisfies

$$
\tilde{X}(\tau, \lambda)-1=\frac{\left(e^{\frac{\tau \lambda}{\sqrt{4+3 \lambda^{2}}}}-1\right)^{2}\left(1+e^{\frac{\tau \lambda}{\sqrt{4+3 \lambda^{2}}}}+e^{\frac{2 \tau \lambda}{\sqrt{4+3 \lambda^{2}}}}\right)}{e^{\frac{3 \tau \lambda}{\sqrt{4+3 \lambda^{2}}}}+e^{\frac{\tau \lambda}{\sqrt{4+3 \lambda^{2}}}}}>0
$$

for $\tau \cdot \lambda \neq 0$.

The second coordinate of the intersection point between the straight line $r$ and $X=0$ is

$$
\tilde{Y}(\tau, \lambda)=\frac{-A(\tau, \lambda)}{C(\tau, \lambda)}=-\frac{e^{-\frac{\lambda \tau}{\sqrt{4+3 \lambda^{2}}}}\left(1+e^{\frac{4 \lambda \tau}{\sqrt{4+3 \lambda^{2}}}}\right) \lambda^{3} \sqrt{4+3 \lambda^{2}}}{\left(-1+e^{\frac{2 \lambda \tau}{\sqrt{4+3 \lambda^{2}}}}\right)\left(3 \lambda^{4}+6 \lambda^{2}+2\right)} .
$$

The slope of the straight line $r$ is

$$
\tilde{m}(\tau, \lambda)=\frac{-B(\tau, \lambda)}{C(\tau, \lambda)}=\frac{\lambda^{2} \sqrt{4+3 \lambda^{2}}\left(e^{\frac{3 \tau \lambda}{\sqrt{4+3 \lambda^{2}}}}+e^{\frac{\tau \lambda}{\sqrt{4+3 \lambda^{2}}}}\right)}{\left(2+6 \lambda^{2}+3 \lambda^{4}\right)\left(\frac{e^{\frac{3 \tau \lambda}{\sqrt{4+3 \lambda^{2}}}}-e^{\frac{\tau \lambda}{\sqrt{4+3 \lambda^{2}}}}}{\lambda}\right)} .
$$

Since inequalities (20) hold, it is clear that $\tilde{m}(\tau, \lambda)$ is positive for $\tau>0$. Therefore, from inequality (22) any solution $(X, Y)$ of system (16) must verify $Y<0$.

Once the principal geometric idea have been presented, we can go ahead with the proof of the lemma.

Item 4 is trivial for the particular case $\lambda=0$. For $\tau \in \bigcup_{k \in \mathbb{N}^{*}}(2 k \pi,(2 k+$ 1) $\pi$ ) and $\lambda \neq 0$, it is obvious that $Y=\sin \tau>0$. Thus $G(\tau, \lambda)$ must be positive and statement 4 is proved. 
Regarding item 5 , for the particular case when $\tau=k \pi$ with $k \in \mathbb{N}$, we have $G(k \pi, \lambda)=A(k \pi, \lambda)+(-1)^{k} B(k \pi, \lambda)$. From this equality it is immediate to prove that $G(k \pi, \lambda)=0$ if and only if $\lambda=0$.

Now let us fix $\tau \in \bigcup_{k \in \mathbb{N}}((2 k-1) \pi, 2 k \pi)$. From item 1, it follows that $G(\tau, 0)<0$. On the other hand, since

$$
\tilde{X}_{\infty}(\tau):=\lim _{\lambda \rightarrow+\infty} \tilde{X}(\tau, \lambda)=\frac{1+e^{\frac{4 \tau}{\sqrt{3}}}}{e^{\frac{\tau}{\sqrt{3}}}+e^{\sqrt{3} \tau}}
$$

and

$$
\tilde{Y}_{\infty}(\tau):=\lim _{\lambda \rightarrow+\infty} \tilde{Y}(\tau, \lambda)=-\frac{e^{-\frac{\tau}{\sqrt{3}}}\left(1+e^{\frac{4 \tau}{\sqrt{3}}}\right)}{\sqrt{3}\left(-1+e^{\frac{2 \tau}{\sqrt{3}}}\right)},
$$

it is easy to deduce that the limit straight line $r$ for $\lambda=+\infty$ is located below the unit circle. In fact, it is a direct consequence of the inequality

$$
\left(\frac{1}{\tilde{X}_{\infty}(\tau)}\right)^{2}+\left(\frac{1}{\tilde{Y}_{\infty}(\tau)}\right)^{2}-1=-\frac{\left(-1+e^{\frac{2 \tau}{\sqrt{3}}}\right)^{4}}{\left(1+e^{\frac{4 \tau}{\sqrt{3}}}\right)^{2}}<0 \text { for } \quad \tau \neq 0
$$

So, function $G(\tau, \lambda)$ must be positive for $\lambda$ large enough. Therefore, there exists at least a positive value $\tilde{\lambda}(\tau)$ such that $G(\tau, \tilde{\lambda}(\tau))=0$.

In order to prove the uniqueness of $\tilde{\lambda}(\tau)$ we are going to analyse the derivative of functions $\tilde{X}(\tau, \lambda)$ and $\tilde{m}(\tau, \lambda)$ with respect to $\lambda$. On the one hand, it is immediate to see that the derivative

$$
\frac{\partial \tilde{X}}{\partial \lambda}(\tau, \lambda)=\frac{4 e^{\frac{-\tau \lambda}{\sqrt{4+3 \lambda^{2}}}}\left(e^{\frac{2 \tau \lambda}{\sqrt{4+3 \lambda^{2}}}}-1\right)\left(1+4 e^{\frac{2 \tau \lambda}{\sqrt{4+3 \lambda^{2}}}}+e^{\frac{4 \tau \lambda}{\sqrt{4+3 \lambda^{2}}}}\right) \tau}{\left(1+e^{\frac{2 \tau \lambda}{\sqrt{4+3 \lambda^{2}}}}\right)^{2}\left(4+3 \lambda^{2}\right)^{\frac{3}{2}}}
$$

is positive for $\lambda>0$ and $\tau>0$. On the other hand, the derivative of function 
$\tilde{m}$ with respect to $\lambda$ can be written as

$$
\frac{\partial \tilde{m}}{\partial \lambda}(\tau, \lambda)=\frac{4 \lambda^{2}\left(3\left(\lambda^{2}+1\right)^{2} \sqrt{4+3 \lambda^{2}} \sinh \left(\frac{2 \tau \lambda}{\sqrt{4+3 \lambda^{2}}}\right)-\lambda\left(3 \lambda^{4}+6 \lambda^{2}+2\right) \tau\right)}{\sinh ^{2}\left(\frac{\tau \lambda}{\sqrt{4+3 \lambda^{2}}}\right)\left(4+3 \lambda^{2}\right)\left(3 \lambda^{4}+6 \lambda^{2}+2\right)^{2}}
$$

whose sign is determined by function

$$
h(\tau, \lambda)=\left(3\left(\lambda^{2}+1\right)^{2} \sqrt{4+3 \lambda^{2}} \sinh \left(\frac{2 \lambda \tau}{\sqrt{4+3 \lambda^{2}}}\right)-\lambda\left(3 \lambda^{4}+6 \lambda^{2}+2\right) \tau\right) .
$$

After the change $\tau=(2 \lambda)^{-1} \sqrt{4+3 \lambda^{2}} \tilde{\tau}$, function $h$ takes the form

$$
\frac{1}{2} \sqrt{4+3 \lambda^{2}}\left((6 \sinh \tilde{\tau}-3 \tilde{\tau}) \lambda^{4}+(12 \sinh \tilde{\tau}-6 \tilde{\tau}) \lambda^{2}+(6 \sinh \tilde{\tau}-2 \tilde{\tau})\right)
$$

which is positive for $\tilde{\tau}>0$. Hence, the derivative of $\tilde{m}$ with respect to $\lambda$ is positive for $\lambda>0$.

From this reasoning it has been proved that the value $\tilde{\lambda}(\tau)>0$ is unique. Another direct consequence is that the partial derivative of function $G$ with respect to $\lambda$ has to be positive at $(\tau, \tilde{\lambda}(\tau))$. Using statement 3 it also follows that $G(\tau,-\tilde{\lambda}(\tau))=0$ and $\frac{\partial G}{\partial \lambda}(\tau,-\tilde{\lambda}(\tau))<0$, what concludes the proof of item 5 .

Note that item 5 allows to define a unique function $\tilde{\lambda}: \tau \in \bigcup_{k \in \mathbb{N}}[(2 k-$ 1) $\pi, 2 k \pi] \rightarrow[0,+\infty)$ such that $G(\tau, \tilde{\lambda}(\tau))=0$. Due to the analyticity of function $G$ and to items 1-5, function $\tilde{\lambda}$ is continuous in every interval [(2k1) $\pi, 2 k \pi]$, analytical in every interval $((2 k-1) \pi, 2 k \pi)$ and $\tilde{\lambda}(k \pi)=0$ for all $k \in \mathbb{N}$. Therefore, for every $k \in \mathbb{N}$ there exists at least a value $\tau_{k} \in$ $((2 k-1) \pi, 2 k \pi)$ such that $\tilde{\lambda}^{\prime}\left(\tau_{k}\right)=0$.

The proof of statement 6 is a direct consequence of item 5 .

In order to prove item 7 one must assure, inter alia, the uniqueness of every $\tau_{k}$. For that purpose it is enough to establish that $\tilde{\lambda}^{\prime \prime}(\tau)$ is always negative 
when $\tilde{\lambda}^{\prime}(\tau)=0$. More concretely, taking into account that $\frac{\partial G}{\partial \lambda}(\tau, \tilde{\lambda}(\tau))>0$ and that

$$
\tilde{\lambda}^{\prime \prime}(\tau)=-\left(\frac{\partial^{2} G}{\partial \tau^{2}}(\tau, \tilde{\lambda}(\tau))\right) /\left(\frac{\partial G}{\partial \lambda}(\tau, \tilde{\lambda}(\tau))\right)
$$

when $\tilde{\lambda}^{\prime}(\tau)=0$, it suffices to prove that for $\tau, \lambda>0$ the second derivative $\frac{\partial^{2} G}{\partial \tau^{2}}(\tau, \lambda)$ is positive when $G(\tau, \lambda)$ and $\frac{\partial G}{\partial \tau}(\tau, \lambda)$ vanish.

For $\lambda \neq 0$, system $G(\tau, \lambda)=\frac{\partial G}{\partial \tau}(\tau, \lambda)=0$ can be written as

$$
\left\{\begin{array}{l}
A(\tau, \lambda)+B(\tau, \lambda) \cos \tau+C(\tau, \lambda) \sin \tau=0 \\
\bar{A}(\tau, \lambda)+\bar{B}(\tau, \lambda) \cos \tau+\bar{C}(\tau, \lambda) \sin \tau=0
\end{array}\right.
$$

where functions $A, B$ and $C$ are defined in (17)-(19) and

$$
\begin{aligned}
& \bar{A}(\tau, \lambda)=4 e^{\frac{4 \lambda \tau}{\sqrt{4+3 \lambda^{2}}}} \lambda^{3} \\
& \bar{B}(\tau, \lambda)=-\frac{2}{\lambda} e^{\frac{\lambda \tau}{\sqrt{4+3 \lambda^{2}}}}\left(2 \lambda^{4}+3 \lambda^{2}-e^{\frac{2 \lambda \tau}{\sqrt{4+3 \lambda^{2}}}}\left(3 \lambda^{2}+1\right)+1\right), \\
& \bar{C}(\tau, \lambda)=\frac{2}{\sqrt{4+3 \lambda^{2}}} e^{\frac{\lambda \tau}{\sqrt{4+3 \lambda^{2}}}}\left(-\lambda^{2}+e^{\frac{2 \lambda \tau}{\sqrt{4+3 \lambda^{2}}}}\left(6 \lambda^{4}+11 \lambda^{2}+3\right)-1\right) .
\end{aligned}
$$

The determinant of the coefficient matrix of $(\cos (\tau), \sin (\tau))$ in system (25) is given by

$$
\begin{aligned}
K(\tau, \lambda) & =\operatorname{det}\left(\begin{array}{cc}
B(\tau, \lambda) & C(\tau, \lambda) \\
\bar{B}(\tau, \lambda) & \bar{C}(\tau, \lambda)
\end{array}\right) \\
& =-\frac{4 e^{\frac{2 \lambda \tau}{\sqrt{4+3 \lambda^{2}}}}\left(2 e^{\frac{2 \lambda \tau}{\sqrt{4+3 \lambda^{2}}}}\left(3 \lambda^{4}+5 \lambda^{2}+1\right) \lambda^{4}+\left(-1+e^{\frac{2 \lambda \tau}{\sqrt{4+3 \lambda^{2}}}}\right)^{2}\left(\lambda^{2}+1\right)^{3}\left(3 \lambda^{2}+1\right)\right)}{\lambda^{2}}
\end{aligned}
$$

which is negative for $\lambda \neq 0$ and $\tau>0$. 
Therefore, system (25) can be solved for the trigonometric functions

$$
\begin{aligned}
\sin (\tau) & =2(K(\tau, \lambda))^{-1} e^{\frac{\tau \lambda}{\sqrt{4+3 \lambda^{2}}}}\left(-1+e^{\frac{2 \tau \lambda}{\sqrt{4+3 \lambda^{2}}}}\right) \lambda \sqrt{4+3 \lambda^{2}} \\
& \times\left(2 \lambda^{4}+3 \lambda^{2}+1+2 e^{\frac{2 \tau \lambda}{\sqrt{4+3 \lambda^{2}}}} \lambda^{4}+e^{\frac{4 \tau \lambda}{\sqrt{4+3 \lambda^{2}}}}\left(2 \lambda^{4}+3 \lambda^{2}+1\right)\right), \\
\cos (\tau) & =2(K(\tau, \lambda))^{-1} e^{\frac{\tau \lambda}{\sqrt{4+3 \lambda^{2}}}}\left(1+e^{\frac{2 \tau \lambda}{\sqrt{4+3 \lambda^{2}}}}\right) \lambda^{2} \\
& \times\left(\lambda^{2}+1+e^{\frac{4 \tau \lambda}{\sqrt{4+3 \lambda^{2}}}}\left(\lambda^{2}+1\right)-2 e^{\frac{2 \tau \lambda}{\sqrt{4+3 \lambda^{2}}}}\left(3 \lambda^{4}+6 \lambda^{2}+2\right)\right) .
\end{aligned}
$$

Moreover, when these expressions of $\sin (\tau)$ and $\cos (\tau)$ are substituted in $\frac{\partial^{2} G}{\partial \tau^{2}}$ and after some trivial simplifications, one realises that the sign of this second derivative of $G$ coincides with the sign of

$$
\begin{aligned}
L(\tau, \lambda)= & e^{\frac{8 \tau \lambda}{\sqrt{4+3 \lambda^{2}}}}\left(\lambda^{2}+1\right)^{3}+2 e^{\frac{6 \tau \lambda}{\sqrt{4+3 \lambda^{2}}}}\left(\lambda^{6}-3 \lambda^{2}-1\right) \\
& +2 e^{\frac{4 \tau \lambda}{\sqrt{4+3 \lambda^{2}}}}\left(3 \lambda^{2}+1\right)\left(\lambda^{2}+1\right)^{2}+2 e^{\frac{2 \tau \lambda}{\sqrt{4+3 \lambda^{2}}}}\left(\lambda^{6}-3 \lambda^{2}-1\right)+\left(\lambda^{2}+1\right)^{3} .
\end{aligned}
$$

The change of variable

$$
u=\exp \left(2 \tau \lambda / \sqrt{4+3 \lambda^{2}}\right)>1
$$

transforms function $L$ into the polynomial function

$$
\begin{aligned}
\tilde{L}(u, \lambda)= & \left(u^{4}+2 u^{3}+6 u^{2}+2 u+1\right) \lambda^{6}+\left(3 u^{4}+14 u^{2}+3\right) \lambda^{4}+ \\
& {\left[3\left(u^{2}+1\right)(u-1)^{2}+4 u^{2}\right] \lambda^{2}+\left(u^{2}+1\right)(u-1)^{2} . }
\end{aligned}
$$

Since all coefficients of $\tilde{L}$ are positive for $u>1$, we obtain that $L(\tau, \lambda)>0$ for $\tau, \lambda>0$.

Therefore we can conclude that the second derivative $\frac{\partial^{2} G}{\partial \tau^{2}}(\tau, \tilde{\lambda}(\tau))$ is positive when $\tau, \lambda>0$ and the proof is finished.

Lemma 2 describes the zero set of function $G$ given in (15). This set is formed by infinitely many simple, closed and isolated curves. Function 
(a)

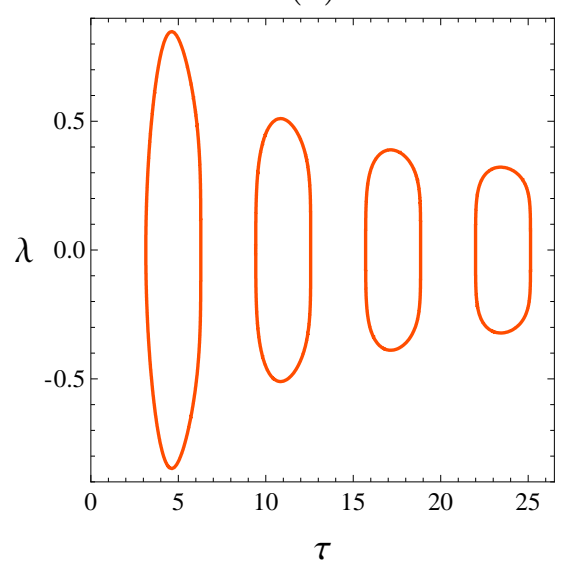

(b)

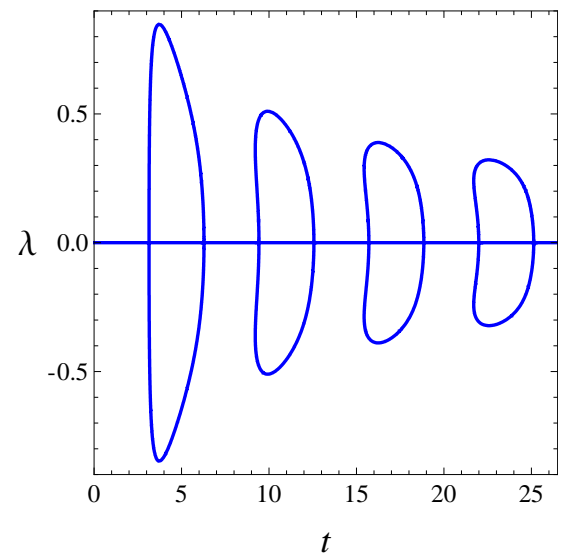

Figure 6: (a) Partial zero set of function $G(\tau, \lambda)$. (b) Partial zero set of function $E(t, \lambda)$.

$G$ is negative in the interior components of theses curves and positive in the exterior component. In Fig. 6(a) we can see several isolated curves of this set. In particular, for each of these curves both kinds of fold points are distinguished. Those given in statement 7 of Lemma 2 correspond to the maximum and minimum values of parameter $\lambda$. On the other hand, the points on the curve where $\lambda=0$ are the fold points given in statement 2. Note that these points are pitchfork singularities for the zero set of function $E$ since $E(t, 0)=0$ for every $t$. In Fig. 6(b), a portion of the zero set of function $E$ is shown. Note that this set corresponds to the image by $t=2 \tau\left(4+3 \lambda^{2}\right)^{-1 / 2}$ of the curves shown in Fig. 6(a), together with the $\lambda=0$ axis.

\section{Study of the inequalities}

We start this section by proving that the condition (6) is satisfied for every $\left(\hat{t}, \hat{\lambda}, \hat{y}_{0}\right)$ that verifies the condition (5). Note that if a triple $\left(t, \lambda, y_{0}\right)=$ 
$\left(\hat{t}, \hat{\lambda}, \hat{y}_{0}\right)$ verifies the condition (5), then the pair $(t, \lambda)$ has to belong to the zero set of function $E$ and $y_{0}$ must have the expression given in (13). If the values $t, \lambda>0$ satisfy $E(t, \lambda)=0$, then from Lemma 2 we have $\sin (\beta t)<0$ and the denominator of (13) is negative. Hence, the sign of $y_{0}$ is the opposite of the sign of its numerator

$$
H(t, \lambda)=\left(2+3 \lambda^{2}\right) \sin (\beta t)+\lambda \sqrt{4+3 \lambda^{2}}\left(e^{\frac{3 \lambda}{2} t}-\cos (\beta t)\right) .
$$

To study this sign it is convenient to consider the change of variable (14) which transforms function $H$ into the function

$$
J(\tau, \lambda)=\left(2+3 \lambda^{2}\right) \sin (\tau)+\lambda \sqrt{4+3 \lambda^{2}}\left(e^{\frac{3 \tau \lambda}{\sqrt{4+3 \lambda^{2}}}}-\cos (\tau)\right) .
$$

Function $J$ verifies some properties that are analogous to those described in Lemma 2 for function $G$, and their proofs are omitted here because they are also similar.

Lemma 3. The following statements about function $J$ hold.

1. If $\tau \in \bigcup_{k \in \mathbb{N}^{*}}(2 k \pi,(2 k+1) \pi)$, then $J(\tau, \lambda)>0$ for all $\lambda>0$.

2. For every $\tau \in \bigcup_{k \in \mathbb{N}}[(2 k-1) \pi, 2 k \pi]$ there exists a unique value $\bar{\lambda}(\tau) \geq 0$ such that $J(\tau, \bar{\lambda}(\tau))=0$. This value $\bar{\lambda}(\tau)$ vanishes at $\tau=k \pi$ for every $k \in \mathbb{N}$. Moreover, if $\tau \in \bigcup_{k \in \mathbb{N}}((2 k-1) \pi, 2 k \pi)$, then $\bar{\lambda}(\tau)>0$ and $\frac{\partial J}{\partial \lambda}(\tau, \bar{\lambda}(\tau))>0$

3. If $\tau \in \bigcup_{k \in \mathbb{N}}[(2 k-1) \pi, 2 k \pi]$, then $\operatorname{sign}(J(\tau, \lambda))=\operatorname{sign}(\lambda-\bar{\lambda}(\tau))$.

4. For every $k \in \mathbb{N}$ there exists a unique pair $\left(\bar{\tau}_{k}, \bar{\lambda}_{k}\right) \in((2 k-1) \pi, 2 k \pi) \times$ $(0,+\infty)$ such that $J\left(\bar{\tau}_{k}, \bar{\lambda}_{k}\right)=\frac{\partial J}{\partial \tau}\left(\bar{\tau}_{k}, \bar{\lambda}_{k}\right)=0$. Moreover, $\frac{\partial^{2} J}{\partial \tau^{2}}\left(\bar{\tau}_{k}, \bar{\lambda}_{k}\right)<$ 0 . 
After Lemma 3 we can state the next proposition.

Proposition 1. Suppose that a triple $\left(t, \lambda, y_{0}\right)=\left(\hat{t}, \hat{\lambda}, \hat{y}_{0}\right)$ verifies condition (5) with $t, \lambda>0$. Then condition (6) holds.

Proof. To prove the thesis of this proposition it is enough to show that every $(\tau, \lambda)$ belonging to the zero set of function $G$ with $\tau, \lambda>0$ satisfies $J(\tau, \lambda)>0$ (see Fig. 7 ), i.e, functions $\tilde{\lambda}$ and $\bar{\lambda}$ defined in lemmas 2 and 3 satisfy $\bar{\lambda}(\tau)<\tilde{\lambda}(\tau)$ for every $\tau \in \bigcup_{k \in \mathbb{N}}((2 k-1) \pi, 2 k \pi)$.

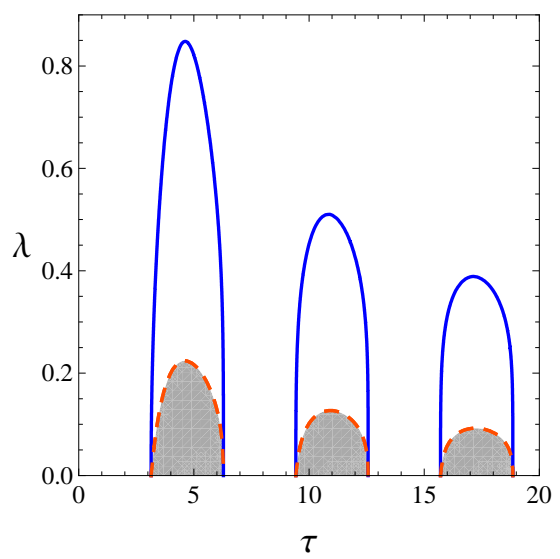

Figure 7: Partial zero set of functions $G(\tau, \lambda)$ (solid line) and $J(t, \lambda)$ (dashed line). The regions where $J(t, \lambda)$ is negative have been shadowed.

To do this, let us consider the straight line

$$
\bar{r} \equiv\left(2+3 \lambda^{2}\right) Y+\lambda \sqrt{4+3 \lambda^{2}}\left(e^{\frac{3 \tau \lambda}{\sqrt{4+3 \lambda^{2}}}}-X\right)=0,
$$

which is obtained by substituting $X=\cos (\tau)$ and $Y=\sin (\tau)$ in (28). We will see that, for every fixed $\tau \geq \pi$ and $\lambda>0$, the straight line $\bar{r}$ is located below the straight line $r$, given in (16), when $Y \leq 0$ and $X \geq-1$ (see Fig. 
8). The idea is to compare the relative position of the straight lines $r$ and $\bar{r}$ when they intersect the straight lines $Y=0$ and $X=-1$.

The first coordinate of the intersection point of straight line $\bar{r}$ with the horizontal axis $Y=0$ is $\bar{X}(\tau, \lambda)=\exp \left(3 \lambda \tau / \sqrt{4+3 \lambda^{2}}\right)$. Respectively, the intersection between $r$ and $Y=0$ is $\tilde{X}(\tau, \lambda)$ given in (21). Note that

$$
\bar{X}(\tau, \lambda)-\tilde{X}(\tau, \lambda)=\frac{-1+e^{\frac{6 \lambda \tau}{\sqrt{4+3 \lambda^{2}}}}}{e^{\frac{\lambda \tau}{\sqrt{4+3 \lambda^{2}}}}+e^{\frac{3 \lambda \tau}{\sqrt{4+3 \lambda^{2}}}}}>0
$$

for $\tau, \lambda>0$ and so $\bar{X}(\tau, \lambda)>\tilde{X}(\tau, \lambda)$ when $\tau, \lambda>0$.

On the other hand, the second coordinate of the intersections points of straight lines $r$ and $\bar{r}$ with $X=-1$ are given by

$$
\tilde{Y}_{1}(\tau, \lambda)=-\frac{e^{-\frac{\lambda \tau}{\sqrt{4+3 \lambda^{2}}}}\left(1+e^{\frac{3 \lambda \tau}{\sqrt{4+3 \lambda^{2}}}}\right) \lambda^{3} \sqrt{4+3 \lambda^{2}}}{\left(-1+e^{\frac{\lambda \tau}{\sqrt{4+3 \lambda^{2}}}}\right)\left(3 \lambda^{4}+6 \lambda^{2}+2\right)}
$$

and

$$
\bar{Y}_{1}(\tau, \lambda)=-\frac{\left(1+e^{\frac{3 \lambda \tau}{\sqrt{4+3 \lambda^{2}}}}\right) \lambda \sqrt{4+3 \lambda^{2}}}{3 \lambda^{2}+2}
$$

respectively. Hence, the sign of $\tilde{Y}_{1}(\tau, \lambda)-\bar{Y}_{1}(\tau, \lambda)$ is given, for $\tau, \lambda>0$, by the sign of function

$$
P(\tau, \lambda)=-\left(3 \lambda^{2}+2\right) \lambda^{2}-e^{\frac{\lambda \tau}{\sqrt{4+3 \lambda^{2}}}}\left(3 \lambda^{4}+6 \lambda^{2}+2\right)+e^{\frac{2 \lambda \tau}{\sqrt{4+3 \lambda^{2}}}}\left(3 \lambda^{4}+6 \lambda^{2}+2\right) .
$$

The change of variable $u=\exp \left(\tau \lambda / \sqrt{4+3 \lambda^{2}}\right)$ transforms function $P$ into function

$$
Q(u, \lambda)=(u-1) u\left(3 \lambda^{4}+6 \lambda^{2}+2\right)-\lambda^{2}\left(3 \lambda^{2}+2\right) .
$$




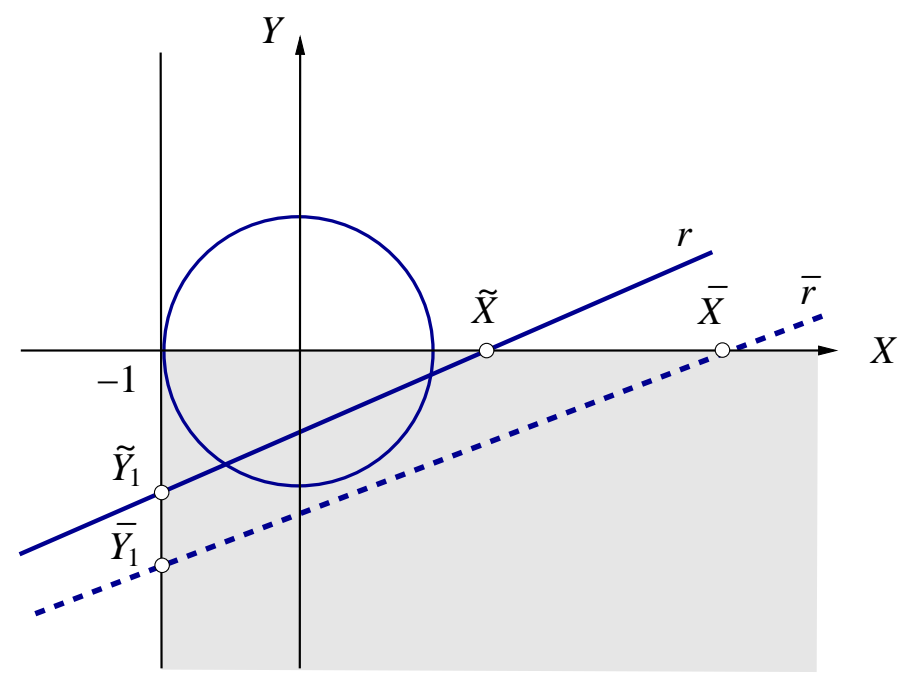

Figure 8: Relative position of the straight lines $r$ and $\bar{r}$ and the points $\bar{X}, \bar{Y}_{1}$ and $\tilde{X}, \tilde{Y}_{1}$. The region $X \geq-1, Y \leq 0$ has been shadowed.

For $\lambda>0$ and $\tau \geq \pi$ we have

$$
e^{\frac{\lambda \tau}{\sqrt{4+3 \lambda^{2}}}} \geq e^{\frac{\lambda \pi}{\sqrt{4+3 \lambda^{2}}}}>e^{\frac{\lambda}{\sqrt{4+3 \lambda^{2}}}}>1+\frac{\lambda}{\sqrt{4+3 \lambda^{2}}}+\frac{\lambda^{2}}{6 \lambda^{2}+8}:=\tilde{u} .
$$

Since $Q(\tilde{u}, \lambda)>0$ and $\frac{\partial Q}{\partial u}(\tilde{u}, \lambda)>0$, we deduce that $Q(u, \lambda)>0$ for $\lambda>0$ and $u \geq \tilde{u}$. Therefore $\bar{Y}_{1}(\tau, \lambda)<\tilde{Y}_{1}(\tau, \lambda)$ and the proof is concluded.

Once we have proved that condition (5) implies inequality (6), we focus on the proof of inequality (7) for $\hat{\lambda}>0$ and $\hat{t} \in[\pi / \hat{\beta}, 2 \pi / \hat{\beta}]$, where $\hat{\beta}=\sqrt{4+3 \hat{\lambda}^{2}} / 2$, that is, when $(\hat{t}, \hat{\lambda})$ belongs to the first $E(t, \lambda)=0$ curve. The next result affirms that when (5), (9) and $\hat{t} \in[\pi / \hat{\beta}, 2 \pi / \hat{\beta}]$ are satisfied, condition (7) holds. So, after the proof of the result we will study condition (9). 
Proposition 2. Let be $\hat{\lambda}>0$ and $\hat{\beta}=\sqrt{4+3 \hat{\lambda}^{2}} / 2$. Assume that $\left(\hat{t}, \hat{\lambda}, \hat{y}_{0}\right)$ satisfies conditions (5), (9) and $\hat{t} \in[\pi / \hat{\beta}, 2 \pi / \hat{\beta}]$. Then, $x\left(t ; \hat{\lambda}, \hat{y}_{0}\right)<0$ for every $t \in(0, \hat{t})$.

Proof. Let us see that, under the hypotheses, if there exist a value $t_{x} \in$ $(0, \hat{t})$ such that $x\left(t_{x} ; \hat{\lambda}, \hat{y}_{0}\right) \geq 0$ then the value $\hat{t}$ must be greater than $2 \pi / \hat{\beta}$.

Remember that the first two equations of system (2) are $\dot{x}=y$ and $\dot{y}=z$ and that (5) implies (6) (see Proposition 1). So, function $x\left(t ; \hat{\lambda}, \hat{y}_{0}\right)$ satisfies $x\left(0 ; \hat{\lambda}, \hat{y}_{0}\right)=x\left(\hat{t} ; \hat{\lambda}, \hat{y}_{0}\right)=0, x\left(t_{x} ; \hat{\lambda}, \hat{y}_{0}\right) \geq 0$ and $\frac{\partial}{\partial t} x\left(0 ; \hat{\lambda}, \hat{y}_{0}\right)=\hat{y}_{0}<$ 0 . Furthermore, function $x\left(t ; \hat{\lambda}, \hat{y}_{0}\right)$ cannot be constant in an open interval because it should correspond to an equilibrium and there are no equilibria at the separation plane.

As a direct conclusion, there exist three values $0<t_{y 1}<t_{y 2}<t_{y 3}<\hat{t}$ such that $y\left(t_{y 1} ; \hat{\lambda}, \hat{y}_{0}\right)<0, y\left(t_{y 2} ; \hat{\lambda}, \hat{y}_{0}\right)>0$ and $y\left(t_{y 3} ; \hat{\lambda}, \hat{y}_{0}\right)<0$. Moreover, condition (9) says that $y\left(\hat{t} ; \hat{\lambda}, \hat{y}_{0}\right) \geq 0$.

It is obvious now that there exist two values $0<t_{z 1}<t_{z 2}<\hat{t}$ where $z\left(t_{z 1} ; \hat{\lambda}, \hat{y}_{0}\right)=z\left(t_{z 2} ; \hat{\lambda}, \hat{y}_{0}\right)=0, y\left(t_{z 1} ; \hat{\lambda}, \hat{y}_{0}\right)>0$ and $y\left(t_{z 2} ; \hat{\lambda}, \hat{y}_{0}\right)<0$. Moreover, from the hypotheses, it holds that $z\left(0 ; \hat{\lambda}, \hat{y}_{0}\right)=z\left(\hat{t} ; \hat{\lambda}, \hat{y}_{0}\right)=0$.

Consider now function $v(t)=-\lambda y\left(t ; \hat{\lambda}, \hat{y}_{0}\right)+z\left(t ; \hat{\lambda}, \hat{y}_{0}\right)$. Since $\hat{\lambda}>0$, from the previous reasoning it is clear that $v(0)>0, v\left(t_{z 1}\right)<0, v\left(t_{z 2}\right)>0$ and $v(\hat{t}) \leq 0$. Therefore, function $v$ vanishes at three values $0<t_{v 1}<t_{v 2}<t_{v 3} \leq$ $\hat{t}$.

Taking into account that the plane $-\lambda y+z=0$ contains the onedimensional unstable manifold $\left\{\mathbf{p}_{-}-\mu\left(1, \lambda, \lambda^{2}\right): \mu \in \mathbb{R}\right\}$ of the equilibrium $\mathbf{p}_{-}$of the linear system $\dot{\mathbf{x}}=A_{-} \mathbf{x}+\mathbf{e}_{3}$, it is obvious that $t_{v 3}-t_{v 1} \geq 2 \pi / \hat{\beta}$. Since $\hat{t}>t_{v 3}-t_{v 1}$, the result is proved. 
Now, we consider condition (9) when $\beta t \in(\pi, 2 \pi)$. Observe that the second component of the solution of system (2) in the half-space $\{x<0\}$ with initial condition $\mathbf{p}_{0}=\left(0, y_{0}, 0\right)$ is given by

$$
\begin{aligned}
y\left(t ; \lambda, y_{0}\right) & =\frac{e^{-\frac{t \lambda}{2}}}{\left(1+3 \lambda^{2}\right) \sqrt{4+3 \lambda^{2}}}\left(\sqrt{4+3 \lambda^{2}}\left(1+\lambda^{2} y_{0}\right) e^{\frac{3 \lambda t}{2}}+\right. \\
& \left.+\sqrt{4+3 \lambda^{2}}\left[\left(1+2 \lambda^{2}\right) y_{0}-1\right] \cos (\beta t)+\lambda\left(y_{0}-3\right) \sin (\beta t)\right) .
\end{aligned}
$$

For any solution $(t, \lambda)$ of equation (12) with $t, \lambda>0$ and for $y_{0}$ given by (13) we have

$y_{0}+\frac{1}{\lambda^{2}}=\frac{2\left(3 \lambda^{4}+4 \lambda^{2}+1\right) \sin (\beta t)}{\sqrt{3 \lambda^{2}+4} \cos (\beta t) \lambda^{5}-e^{\frac{3 t \lambda}{2}} \sqrt{3 \lambda^{2}+4} \lambda^{5}+\left(3 \lambda^{4}+6 \lambda^{2}+2\right) \sin (\beta t) \lambda^{2}}>0$,

that is, condition (8) is satisfied.

Note that, for $\beta t \in(\pi, 3 \pi / 2]$, inequality (30) implies that $y\left(t ; \lambda, y_{0}\right)>0$. Thus, any solution $(t, \lambda)$ of equation (12) with $\beta t \in(\pi, 3 \pi / 2]$ and $\lambda>0$ corresponds to a RP2-orbit. For the rest of the interval, $\beta t \in(3 \pi / 2,2 \pi)$, the conclusion is not so easy because, as we will see below, there exists a point where the sign of $y\left(t ; \lambda, y_{0}\right)$ changes. Obviously, the orbit corresponding to such a point intersects the separation plane at the tangency line $\{x=0, y=$ $0\}$. It is then natural to analyze the orbits that go into $\{x<0\}$ through $\operatorname{Fix}(\mathbf{R})$ and, after that, have a first intersection with the separation plane at the tangency line.

By integrating the linear system corresponding to the half-space $\{x<0\}$, a point $\left(0, y_{0}, 0\right)$ is mapped onto a point at $\{x=0, y=0\}$ if there exist $t, \lambda>0$ such that

$$
\left\{\begin{array}{c}
M_{1}\left(t, \lambda, y_{0}\right)=0 \\
M_{2}\left(t, \lambda, y_{0}\right)=0
\end{array}\right.
$$


where

$$
\begin{aligned}
M_{1}\left(t, \lambda, y_{0}\right) & =\left(\left(\lambda^{2}+1\right)\left(\lambda^{2} y_{0}+1\right) e^{t \lambda}-3 \lambda^{2}-1\right) \sqrt{3 \lambda^{2}+4} e^{\frac{t \lambda}{2}} \\
& +\lambda\left(\left(\lambda^{2}+1\right)\left(3 \lambda^{2}+2\right) y_{0}-2\right) \sin (\beta t) \\
& -\lambda^{2}\left(\left(\lambda^{2}+1\right) y_{0}-2\right) \sqrt{3 \lambda^{2}+4} \cos (\beta t)
\end{aligned}
$$

and

$M_{2}\left(t, \lambda, y_{0}\right)=\left(\left(\lambda^{2} y_{0}+1\right) e^{\frac{3}{2} t \lambda}+\left(\left(2 \lambda^{2}+1\right) y_{0}-1\right) \cos (\beta t)\right) \sqrt{3 \lambda^{2}+4}-\lambda\left(y_{0}-3\right) \sin (\beta t)$.

The coefficient of $y_{0}$ in $M_{1}\left(t, \lambda, y_{0}\right)$ is given by $\lambda\left(\lambda^{2}+1\right) H(t, \lambda)$, where $H$ is defined in (27). If function $H$ does not vanish for a pair $(t, \lambda)$, then $y_{0}$ can be solved from the first equation of system (31),

$y_{0}(t, \lambda)=\frac{2 \lambda \sin (\beta t)-\left(2 \lambda^{2} \cos (\beta t)+\left(\left(\lambda^{2}+1\right) e^{t \lambda}-3 \lambda^{2}-1\right) e^{\frac{t \lambda}{2}}\right) \sqrt{3 \lambda^{2}+4}}{\lambda\left(\lambda^{2}+1\right) H(t, \lambda)}$.

By substituting this expression in the second equation of (31), one obtains $M(t, \lambda)=0$, where

$$
\begin{aligned}
M(t, \lambda) & =-e^{\frac{t \lambda}{2}}\left(-1+e^{t \lambda}\right)\left(6 \lambda^{4}+11 \lambda^{2}+4\right) \cos (t \beta) \\
& +\left(1+e^{t \lambda}\right) \lambda\left(\left(-1+e^{t \lambda}\right) \lambda\left(4+3 \lambda^{2}\right)+e^{\frac{t \lambda}{2}} \sqrt{4+3 \lambda^{2}} \sin (t \beta)\right) .
\end{aligned}
$$

To explore the zero set of function $M$ for $\beta t \in[3 \pi / 2,2 \pi]$ we consider the function $N$ defined as

$$
N(\tau, \lambda)=S\left(\frac{2 \tau}{\sqrt{4+3 \lambda^{2}}}, \lambda\right)
$$

where

$$
S(t, \lambda)= \begin{cases}\frac{M(t, \lambda)}{\lambda} & \text { if } \lambda \neq 0, \\ -4 t \cos (t)+4 \sin (t) & \text { if } \quad \lambda=0 .\end{cases}
$$

The next lemma summarize several features of the zero set of function $N$ for $\tau \in[3 \pi / 2,2 \pi]$ and $\lambda>0$. 
Lemma 4. Function $N$ satisfies the following properties.

1. Given $\tau \in[3 \pi / 2,2 \pi]$, there exist a unique value $\lambda^{*}(\tau)>0$ such that $N\left(\tau, \lambda^{*}(\tau)\right)=0$. Moreover, the derivative $\frac{\partial N}{\partial \lambda}$ verifies $\frac{\partial N}{\partial \lambda}\left(\tau, \lambda^{*}(\tau)\right)>0$.

2. If $\tau \in[3 \pi / 2,2 \pi]$ and $\lambda>0$ then $\operatorname{sign}(N(\tau, \lambda))=\operatorname{sign}\left(\lambda-\lambda^{*}(\tau)\right)$.

3. Function $\lambda^{*}:[3 \pi / 2,2 \pi] \rightarrow(0,+\infty)$ is analytical and has a unique critical value $\tau_{C} \in(3 \pi / 2,2 \pi)$. Moreover, this critical value $\tau_{C}$ corresponds to the maximum value of function $\lambda^{*}$ in the interval $[3 \pi / 2,2 \pi]$.

4. The critical value $\tau_{C}$ satisfies $G\left(\tau_{C}, \lambda^{*}\left(\tau_{C}\right)\right)=0$, where $G$ is defined in (15).

Proof. We will only prove statement 4 because the rest of items can be proved as in Lemma 2.

The pair $\left(\tau_{C}, \lambda^{*}\left(\tau_{C}\right)\right)$ is the unique solution of system

$$
\left\{\begin{array}{l}
N(\tau, \lambda)=0, \\
\frac{\partial N}{\partial \tau}(\tau, \lambda)=0,
\end{array}\right.
$$

in $(3 \pi / 2,2 \pi) \times(0,+\infty)$.

Taking first order derivative of function $N$ with respect to $\tau$, system (34) for $\lambda>0$ can be written as

$$
\left\{\begin{array}{l}
-e^{\frac{\lambda \tau}{\sqrt{4+3 \lambda^{2}}}}\left(-1+e^{\frac{2 \lambda \tau}{\sqrt{4+3 \lambda^{2}}}}\right)\left(6 \lambda^{4}+11 \lambda^{2}+4\right) \cos \tau+ \\
\left(1+e^{\frac{2 \lambda \tau}{\sqrt{4+3 \lambda^{2}}}}\right) \lambda\left(\left(-1+e^{\frac{2 \lambda \tau}{\sqrt{4+3 \lambda^{2}}}}\right) \lambda\left(4+3 \lambda^{2}\right)+e^{\frac{\lambda \tau}{\sqrt{4+3 \lambda^{2}}}} \sqrt{3 \lambda^{2}+4} \sin \tau\right)=0, \\
2 e^{\frac{3 \lambda \tau}{\sqrt{4+3 \lambda^{2}}}} \lambda^{3}\left(4+3 \lambda^{2}\right)-\lambda\left(4+3 \lambda^{2}\right)\left(-\lambda^{2}+e^{\frac{2 \lambda \tau}{\sqrt{4+3 \lambda^{2}}}}\left(3 \lambda^{2}+1\right)-1\right) \cos \tau+ \\
\sqrt{4+3 \lambda^{2}}\left(-3 \lambda^{4}-5 \lambda^{2}+e^{\frac{2 \lambda \tau}{\sqrt{4+3 \lambda^{2}}}}\left(3 \lambda^{4}+7 \lambda^{2}+2\right)-2\right) \sin \tau=0 .
\end{array}\right.
$$


Adding $-\lambda$ times the first equation to $\exp \left(\lambda \tau / \sqrt{4+3 \lambda^{2}}\right)$ times the second one, the equation

$$
\begin{aligned}
& \left(1+e^{\frac{4 \lambda \tau}{\sqrt{4+3 \lambda^{2}}}}\right) \lambda^{3}\left(4+3 \lambda^{2}\right)-e^{\frac{\lambda \tau}{\sqrt{4+3 \lambda^{2}}}}\left(1+e^{\frac{2 \lambda \tau}{\sqrt{3 \lambda^{2}+4}}}\right) \lambda^{3}\left(3 \lambda^{2}+4\right) \cos \tau+ \\
& e^{\frac{\lambda \tau}{\sqrt{4+3 \lambda^{2}}}}\left(-1+e^{\frac{2 \lambda \tau}{\sqrt{4+3 \lambda^{2}}}}\right) \sqrt{4+3 \lambda^{2}}\left(3 \lambda^{4}+6 \lambda^{2}+2\right) \sin (\tau)=0
\end{aligned}
$$

is obtained.

It is direct to see that this last equation is equivalent to $G(\tau, \lambda)=0$ for $\lambda, \tau>0$ and so the proof is finished.

In Figure 9 we can see the zero set of function $G(\tau, \lambda)$ for $\tau \in(\pi, 2 \pi)$ and the zero set of function $N(\tau, \lambda)$ for $\tau \in(3 \pi / 2,2 \pi)$. It can be observed that both curves intersect at a unique point that, moreover, corresponds to the maximum of function $\lambda^{*}(\tau)$ defined in Lemma 4 .

After Proposition 2, we saw that any solution $(t, \lambda)$ of equation (12) with $\tau=\beta t \in(\pi, 3 \pi / 2]$ and $\lambda>0$ corresponds to a RP2-orbit, because $y\left(t ; \lambda, y_{0}\right)>0$ holds. From Lemma 4 it is easy to see that this is also true for $\tau=\beta t \in\left(3 \pi / 2, \tau_{C}\right]$ and $\lambda>0$ because $\tau_{C}$ is the only value for which $y\left(t ; \lambda, y_{0}\right)=0$. Moreover, the period of these RP2-orbits is less than $4 \pi$ since $t=2 \tau\left(4+3 \lambda^{2}\right)^{-1 / 2} \leq \tau$. This is summarized in the following result.

Proposition 3. Let $\tau_{C} \in(3 \pi / 2,2 \pi)$ the critical value defined in Lemma 4 and let $\tilde{\lambda}$ be the function defined in Lemma 2. If $\tau \in\left(\pi, \tau_{C}\right)$, then system (2) has a RP2-orbit for $\lambda=\tilde{\lambda}(\tau)$ whose period is less than $4 \pi$.

\section{Last details of the proof}

This section is devoted to two remaining details that must be checked to give a complete proof of Theorem 1 . On the one hand, we must see that 


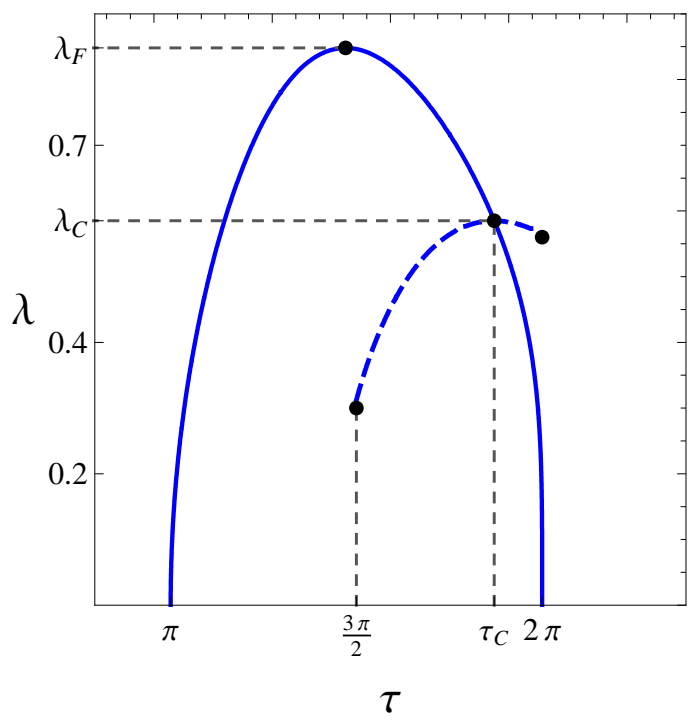

Figure 9: Partial zero sets of functions $G$ (solid line) and $N$ (dashed line). The intersection between these two curves is the point $\left(\tau_{C}, \lambda_{C}\right)$ where function $\lambda^{*}$ reaches its maximum value.

there not exist other RP2-orbits with period less than $4 \pi$ apart from the ones obtained in Proposition 3. On the other hand, we will see that the point $\left(\tau_{1}, \lambda_{1}\right)$ defined in item 7 of Lemma 2 satisfies the inequality $\tau_{1}<\tau_{C}$ and so it corresponds to a saddle-node bifurcation of RP2-orbits of system (2).

For the first thing, we are going to prove that the RP2-orbits that might correspond to the zero curves of $G$ with $\tau \geq 3 \pi$ must have period greater than $4 \pi$. This can be done if we see that all these curves are included in the horizontal half-stripe $\Gamma_{\tau}=\{(\tau, \lambda) \in[3 \pi,+\infty) \times(-1,1)\}$, whose image by the change $t=2 \tau\left(4+3 \lambda^{2}\right)^{-1 / 2}$ is included in $\{(t, \lambda) \in(2 \pi,+\infty) \times(-1,1)\}$. The following proposition is devoted to prove it. 
Proposition 4. The image of the set $\left\{(\tau, \lambda) \in \mathbb{R}^{2}: G(\tau, \lambda)=0, \tau \geq 3 \pi\right\}$ by the change $t=2 \tau\left(4+3 \lambda^{2}\right)^{-1 / 2}$ is included in $\Gamma_{t}=\{(t, \lambda) \in(2 \pi,+\infty) \times$ $(-1,1)\}$.

Proof. Let us begin by proving that function $G$ is positive for $\tau \geq 3 \pi$ and $\lambda \geq 1$. To do this, by following the proof of Lemma 2 , it is enough to see that the straight line $r$ defined in (16) is located below the unit circle for $\tau \geq 3 \pi$ and $\lambda \geq 1$. Since functions $\tilde{X}$ and $\tilde{Y}$ given in (21) and (23) are increasing with respect to $\lambda$ when $\tau$ is fixed, it suffices to prove that the straight line $r$ is located below the unit circle for $\tau \geq 3 \pi$ and $\lambda=1$.

When $\lambda=1$, the intersection points of straight line $r$ with the horizontal and vertical axis are given by

$$
\tilde{X}(\tau, 1)=\frac{1+e^{\frac{4 \tau}{\sqrt{7}}}}{e^{\frac{\tau}{\sqrt{7}}}+e^{\frac{3 \tau}{\sqrt{7}}}}
$$

and

$$
\tilde{Y}(\tau, 1)=-\frac{\sqrt{7} e^{-\frac{\tau}{\sqrt{7}}}\left(1+e^{\frac{4 \tau}{\sqrt{7}}}\right)}{11\left(-1+e^{\frac{2 \tau}{\sqrt{7}}}\right)},
$$

respectively.

Note that the straight line $r$ is located below the unit circle if $\tilde{X}(\tau, 1)^{-2}+$ $\tilde{Y}(\tau, 1)^{-2}-1<0$. Since

$$
\frac{1}{\tilde{X}(\tau, 1)^{2}}+\frac{1}{\tilde{Y}(\tau, 1)^{2}}-1=-\frac{\left(-1+e^{\frac{2 \tau}{\sqrt{7}}}\right)^{2}\left(7-114 e^{\frac{2 \tau}{\sqrt{7}}}+7 e^{\frac{4 \tau}{\sqrt{7}}}\right)}{7\left(1+e^{\frac{4 \tau}{\sqrt{7}}}\right)^{2}}
$$

it is necessary to prove that the function $p(\tau)=7-114 e^{\frac{2 \tau}{\sqrt{7}}}+7 e^{\frac{4 \tau}{\sqrt{7}}}$ is positive for $\tau \geq 3 \pi$. But, it is trivial because the change of variable $s=e^{\frac{2 \tau}{\sqrt{7}}}$ transforms function $p$ into the quadratic polynomial $q(s)=7 s^{2}-114 s+7$ which is clearly positive for $s \geq \exp (6 \pi / \sqrt{7})$. 
As a direct consequence, the set $\left\{(\tau, \lambda) \in \mathbb{R}^{2}: G(\tau, \lambda)=0, \tau \geq 3 \pi\right\}$ is contained in the horizontal half-stripe $\Gamma_{\tau}=\{(\tau, \lambda) \in[3 \pi,+\infty) \times(-1,1)\}$.

On the other hand, the image of every half-straight line $\tau=$ constant, $\lambda>0$ by means of the transformation $t=2 \tau\left(4+3 \lambda^{2}\right)^{-1 / 2}$ is a curve that decreases with respect to $\lambda$. So, since the image of the point $(\tau, \lambda)=(3 \pi, 1)$ belongs to $\Gamma_{t}=\{(t, \lambda) \in(2 \pi,+\infty) \times(-1,1)\}$, the image of $\Gamma_{\tau}$ is included in $\Gamma_{t}$ and the proof is concluded.

Note that in the proof of Proposition 4 it is not necessary to know the sign of $G$ when $\tau \in[0,3 \pi)$ and $\lambda \geq 1$, but it is possible to see that it is also positive.

Now we are going to prove that every solution $(\bar{\tau}, \bar{\lambda})$, with $\bar{\tau}, \bar{\lambda}>0$, of system

$$
\left\{\begin{array}{l}
G(\tau, \lambda)=0, \\
N(\tau, \lambda)=0,
\end{array}\right.
$$

verifies $\tilde{\lambda}^{\prime}(\bar{\tau})<0$. In this way, function $\tilde{\lambda}$ is decreasing for $\tau=\tau_{C}$ and so the value $\tau_{1}$ defined in item 7 of Lemma 2 is less than $\tau_{C}$. Therefore, the fold point $\left(\tau_{1}, \lambda_{1}\right)$ gives a RP2-orbit and it corresponds to a saddle-node bifurcation of periodic orbits of system (2).

Proposition 5. System (2) undergoes a saddle-node bifurcation of periodic orbits for $\lambda=\lambda_{1}$.

Proof. As we have just said, to prove the thesis of the proposition it suffices to prove that every solution $(\bar{\tau}, \bar{\lambda})$ of system $(35)$ satisfy $\tilde{\lambda}^{\prime}(\bar{\tau})<0$.

To do this, let us begin by proving that function

$$
W(\tau, \lambda)=\left(1+\lambda^{2}\right)^{2}+\left(1+\lambda^{2}\right)^{2} e^{\frac{4 \lambda \tau}{\sqrt{4+3 \lambda^{2}}}}-2\left(1+3 \lambda^{2}+\lambda^{4}\right) e^{\frac{2 \lambda \tau}{\sqrt{4+3 \lambda^{2}}}}
$$


is positive for $\lambda>0$ and $\tau \geq \pi$.

The change of variable given by (26) transforms function $W$ into the function

$$
\hat{W}(u, \lambda)=\left(1+\lambda^{2}\right)^{2}+\left(1+\lambda^{2}\right)^{2} u^{2}-2 u\left(1+3 \lambda^{2}+\lambda^{4}\right) .
$$

For $\lambda>0$ and $\tau \geq \pi$, we have

$$
e^{\frac{2 \lambda \tau}{\sqrt{4+3 \lambda^{2}}}} \geq e^{\frac{2 \lambda \pi}{\sqrt{4+3 \lambda^{2}}}}>e^{\frac{4 \lambda}{\sqrt{4+3 \lambda^{2}}}}>1+\frac{4 \lambda}{\sqrt{4+3 \lambda^{2}}}:=\hat{u}
$$

It is easy to see that $\hat{W}(\hat{u}, \lambda)>0$ and $\frac{\partial \hat{W}}{\partial u}(\hat{u}, \lambda)>0$. Therefore $\hat{W}(u, \lambda)>0$ for $\lambda>0, u \geq \hat{u}$ and $W(\tau, \lambda)>0$ for $\lambda>0, \tau \geq \pi$.

Hence, function

$$
T(\tau, \lambda)=\left(1+3 \lambda^{2}\right) W(\tau, \lambda)+\left(2 \lambda^{2}+4 \lambda^{4}\right) e^{\frac{2 \lambda \tau}{\sqrt{4+3 \lambda^{2}}}}
$$

is positive for $\lambda>0$ and $\tau \geq \pi$.

For $\lambda, \tau>0$, system (35) can be written in the form

$$
\left\{\begin{array}{l}
\left(1+e^{\frac{4 \lambda \tau}{\sqrt{4+3 \lambda^{2}}}}\right) \lambda^{3}\left(4+3 \lambda^{2}\right)-e^{\frac{\lambda \tau}{\sqrt{4+3 \lambda^{2}}}}\left(1+e^{\frac{2 \lambda \tau}{\sqrt{4+3 \lambda^{2}}}}\right) \lambda^{3}\left(4+3 \lambda^{2}\right) \cos (\tau)+ \\
e^{\frac{\lambda \tau}{\sqrt{4+3 \lambda^{2}}}}\left(-1+e^{\frac{2 \lambda \tau}{\sqrt{4+3 \lambda^{2}}}}\right) \sqrt{4+3 \lambda^{2}}\left(3 \lambda^{4}+6 \lambda^{2}+2\right) \sin (\tau)=0, \\
-e^{\frac{\lambda \tau}{\sqrt{4+3 \lambda^{2}}}}\left(-1+e^{\frac{2 \lambda \tau}{\sqrt{3+3 \lambda^{2}}}}\right)\left(6 \lambda^{4}+11 \lambda^{2}+4\right) \cos (\tau)+ \\
\left(1+e^{\frac{2 \lambda \tau}{\sqrt{3+3 \lambda^{2}}}}\right) \lambda\left(\left(-1+e^{\frac{2 \lambda \tau}{\sqrt{4+3 \lambda^{2}}}}\right) \lambda\left(4+3 \lambda^{2}\right)+\sqrt{4+3 \lambda^{2}} e^{\frac{\lambda \tau}{\sqrt{4+3 \lambda^{2}}}} \sin (\tau)\right)=0 .
\end{array}\right.
$$

By solving this system for $\cos (\tau)$ and $\sin (\tau)$ we obtain a unique solution given by 


$$
\begin{aligned}
& \sin (\tau)=-\frac{e^{-\frac{\lambda \tau}{\sqrt{4+3 \lambda^{2}}}}\left(-1+e^{\frac{2 \lambda \tau}{\sqrt{4+3 \lambda^{2}}}}\right) \lambda^{3} \sqrt{4+3 \lambda^{2}}\left(-2 e^{\frac{2 \lambda \tau}{\sqrt{4+3 \lambda^{2}}}} \lambda^{2}+1+\lambda^{2}+e^{\frac{4 \lambda \tau}{\sqrt{4+3 \lambda^{2}}}}\left(\lambda^{2}+1\right)\right)}{2 T(\tau, \lambda)} \\
& \cos (\tau)=\frac{e^{-\frac{\lambda \tau}{\sqrt{4+3 \lambda^{2}}}}\left(1+e^{\frac{2 \lambda \tau}{\sqrt{4+3 \lambda^{2}}}}\right) \lambda^{2}\left(3 \lambda^{4}+5 \lambda^{2}+2+e^{\frac{4 \lambda \tau}{\sqrt{4+3 \lambda^{2}}}}\left(3 \lambda^{4}+5 \lambda^{2}+2\right)-2 e^{\frac{2 \lambda \tau}{\sqrt{4+3 \lambda^{2}}}}\left(3 \lambda^{4}+6 \lambda^{2}+2\right)\right)}{2 T(\tau, \lambda)}
\end{aligned}
$$

From statement 5 of Lemma 2 we know that $\frac{\partial G}{\partial \lambda}(\tau, \tilde{\lambda}(\tau))>0$ and so the sign of

$$
\tilde{\lambda}^{\prime}(\tau)=-\left(\frac{\partial G}{\partial \tau}(\tau, \tilde{\lambda}(\tau))\right) /\left(\frac{\partial G}{\partial \lambda}(\tau, \tilde{\lambda}(\tau))\right)
$$

is determined by the sign of the derivative $\frac{\partial G}{\partial \tau}(\tau, \tilde{\lambda}(\tau))$.

By substituting the expressions of $\cos (\tau)$ and $\sin (\tau)$ given by (36) in the derivative $\frac{\partial G}{\partial \tau}(\tau, \tilde{\lambda}(\tau))$ and after some trivial computations it is obtained $\frac{\partial G}{\partial \tau}(\tau, \tilde{\lambda}(\tau))=2\left(-1+e^{\frac{4 \tilde{\lambda}(\tau) \tau}{\sqrt{4+3 \tilde{\lambda}(\tau)^{2}}}}\right) \tilde{\lambda}(\tau)\left(3 \tilde{\lambda}(\tau)^{4}+4 \tilde{\lambda}(\tau)^{2}+1\right) \frac{W(\tau, \tilde{\lambda}(\tau))}{T(\tau, \tilde{\lambda}(\tau))}>0$ and so the conclusion follows.

Note that $\lambda_{1}$ is the value $\lambda_{F}$ given in Theorem 1 and that $\lambda_{C}$ stands for $\tilde{\lambda}\left(\tau_{C}\right)$.

\section{Some comments about other RP2-orbits}

Theorem 1 is devoted to RP2-orbits whose period is less than $4 \pi$. Remember that the procedure to analyze those orbits began with the study of the zero set of function $E$ given in Eq. (12). After that, in Section 3, we restricted the period to prove the inequalities that RP2-orbits must satisfy. Nevertheless, from Lemma 2 (and Figure 6(b)), we know that there exist infinitely many curves belonging to the zero set of function $E$. These curves, 
except the first one, correspond, in case of being periodic orbits, to higher periods. In this last section we are going to give some numerical results about when the points of these curves correspond or not with RP2-orbits.

In Figure 10(a), a RP2-orbit with period greater than $4 \pi$ is shown. A such orbit is characterized by some extra loops around the one-dimensional manifold between two consecutive intersections with the separation plane and, as we can see in Figure 10(b), a natural way for them to disappear is the existence of tangencies with the separation plane $\left(\hat{p}_{1}\right.$ and $\left.\hat{p}_{3}\right)$. Some results about tangencies and their importance can be found in [15].

(a)

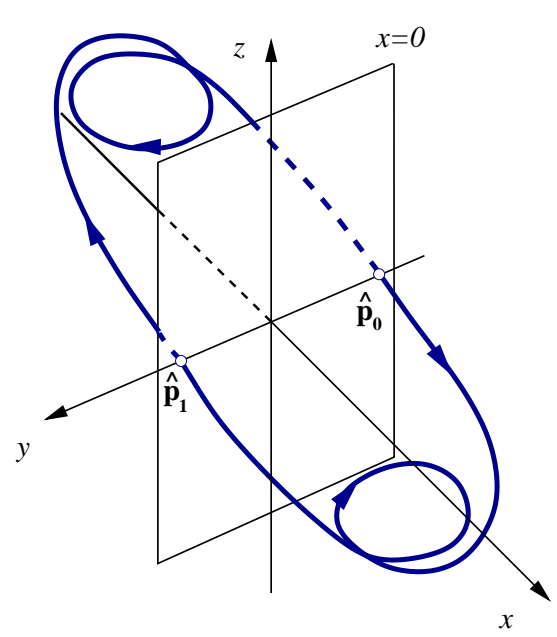

(b)

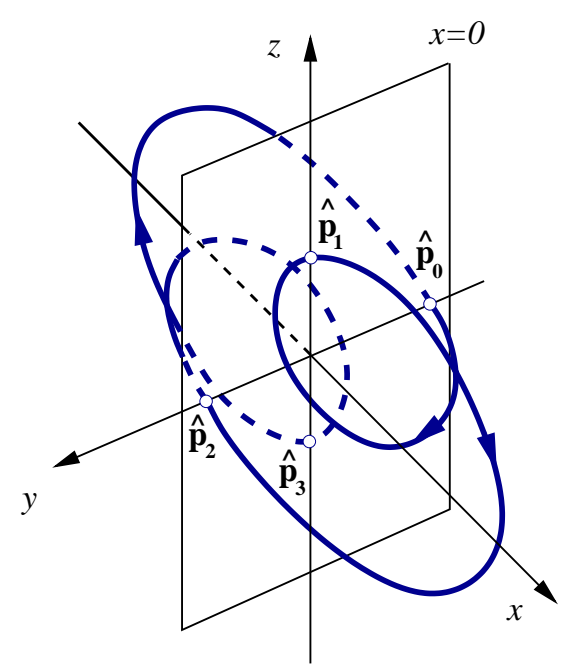

Figure 10: (a) RP2-orbit of system (2) with period greater than $4 \pi$. (b) Reversible periodic orbit of system (2) which has exactly two transversal and two non transversal intersections with $\{x=0\}$.

In the particular case of the second curve of the zero set of $E$ (that is, the first one of Figure 6(b) with period greater than $4 \pi$ ), it has been checked 
that there exist only a piece that gives RP2-orbits (similar to those shown in Figure 10(a)). The end points of this piece are approximately $(t, \lambda)=$ $(9.28741,0.442641)$ and $(t, \lambda)=(11.27502,0.424947)$ and they correspond to non-transversal tangencies (as those shown in Figure 10(b)). On the other hand, after testing some of them, the rest of curves do not seem to give any RP2-orbits. A plausible reason may be that the extra loops are big enough to intersect the separation plane and, therefore, condition (7) cannot be fulfilled.

In Figure 11 the first three curves of the zero set of function $E$ have been numerically drawn. The solid line correspond to points that give RP2-orbits while the dotted line do not. The zero set of function $M$ given in (32) is also shown as a dashed line in Figure 11. From item 4 of Lemma 4 it is deduced that the maximum points of the zero set of function $M$ are located over the zero set of $E$. Moreover, in the case of the first curve this point was the end point of the curve of RP2-orbits, given by a transversal tangency with the separation plane. The maximum points of the zero set of $M$ at the other curves do not have this significance, because they do not correspond to RP2-orbits.

A last remark to finish the section. The maximum point of the second curve of the zero set of function $E$ belongs to the piece where RP2-orbits exist. So, it is a fold bifurcation of periodic orbits.

[1] A. Algaba, E. Freire, E. Gamero and A. J. Rodriguez-Luis, Resonances of periodic orbits in Rssler system in presence of a triple-zero bifurcation, Internat. J. Bifur. Chaos Appl. Sci. Engrg. 17 (2007) 1997-2008.

[2] A. Arneodo, P. Coullet and C. Tresser, Oscillators with chaotic behavior: 


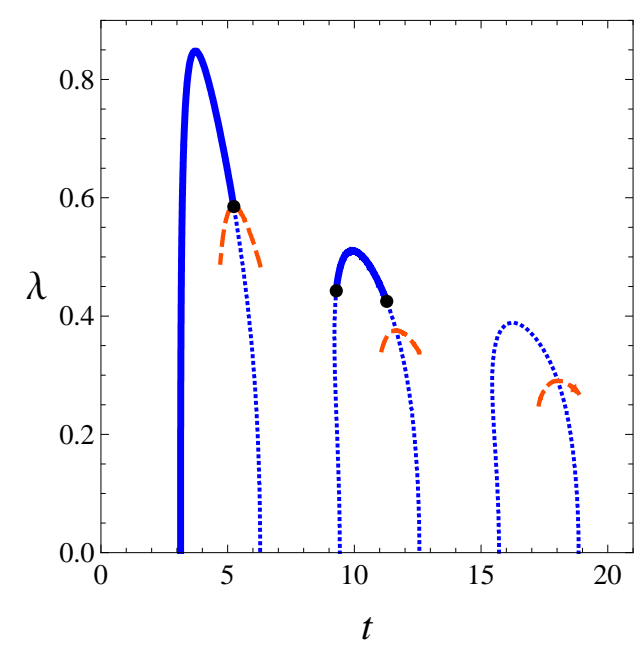

Figure 11: Partial zero sets of function E (solid and dotted lines) and function M (dashed line). Solid lines correspond to RP2-orbits while dotted lines do not.

an illustration of a theorem by Shil'nikov, J. Statist. Phys. 27 (1982) $171-182$.

[3] V. Carmona, F. Fernández-Sánchez and A. E. Teruel, Existence of a reversible T-point heteroclinic cycle in a piecewise linear version of the Michelson system, SIAM J. Appl. Dyn. Syst. 7 (2008) 1032-1048.

[4] V. Carmona, F. Fernández-Sánchez E. García-Medina and A. E. Teruel, Existence of homoclinic connections in continuous piecewise linear systems, Chaos 20 (2010) 013124.

[5] V. Carmona, E. Freire, E. Ponce, J. Ros, F. Torres, Limit cycle bifurcation in $3 \mathrm{D}$ continuous piecewise linear systems with two zones. Application to Chua's circuit, Internat. J. Bifur. Chaos Appl. Sci. Engrg. 15 (2005) 3153-3164. 
[6] F. Dumortier, S. Ibañez and H. Kokubu, New aspects in the unfolding of the nilpotent singularity of codimension three, Dyn. Syst.,16 (2001), $63-95$.

[7] E. Freire, E. Gamero, A. J. Rodriguez-Luis and A. Algaba, A note on the triple-zero linear degeneracy: normal forms, dynamical and bifurcation behaviors of an unfolding, Internat. J. Bifur. Chaos Appl. Sci. Engrg. 12 (2002) 2799-2820.

[8] E. Freire, E. Ponce, J. Ros, Limit cycle bifurcation from center in symmetric piecewise-linear systems, Internat. J. Bifur. Chaos Appl. Sci. Engrg. 9 (1999) 895-907.

[9] E. Freire, E. Ponce, F. Torres, Hopf-like bifurcations in planar piecewise linear systems, Publ. Mat. 41 (1997) 135-148.

[10] P. Kent and J. Elgin, Noose bifurcation of periodic orbits, Nonlinearity 4, (1991) 1045-1061.

[11] H. Kokubu, D. Wilczak, P. Zgliczyński, Rigorous verification of cocoon bifurcations in the Michelson system, Nonlinearity 20 (2007) 2147-2174.

[12] Y. Kuramoto and T. Tsuzuki, Persistent propagation of concentration waves in dissipative media far for thermal equilibrium, Prog. Theoret. Phys. 55 (1976) 356-369.

[13] Yu. A. Kuznetsov, Elements of Applied Bifurcation Theory, SpringerVerlag, New York, 2004. 
[14] J. Lamb, M. A. Teixeira, K. N. Webster, Heteroclinic bifurcations near Hopf-zero bifurcation in reversible vector fields in $\mathbb{R}^{3}$, J. Differ. Equations, 219 (2005) 78-115.

[15] C.M. Lee, P.J. Collins, B. Krauskopf and H.M. Osinga, Tangency bifurcations of global Poincaré maps, SIAM Journal on Applied Dynamical Systems 7(3) (2008) 712-754.

[16] J. Llibre and A. E. Teruel, Existence of Poincaré maps in piecewise linear differential systems in $\mathbb{R}^{n}$, Internat. J. Bifur. Chaos Appl. Sci. Engrg. 8 (2004) 2843-2851.

[17] J. Llibre, X. Zhang, On the Hopf-zero bifurcation of the Michelson system, Nonlinear anal-real 12 (2011) 1650-1653.

[18] D. Michelson, Steady solutions of the Kuramoto-Sivashinsky equation, Phys. D 19 (1986) 89-111.

[19] L. P. Shil'nikov, A case of the existence of a denumerable set of periodic motions, Sov. Math. Dokl. 6 (1965) 163-166.

[20] C. Tresser, About some theorems by L. P. Shil'nikov, Ann. Inst. H. Poincaré Phys. Théor. 40 (1984) 441-461.

[21] W. C. Troy, The existence of steady solutions of the KuramotoSivashinsky equation, J. Differ. Equations 82 (1989) 269-313.

[22] K. N. Webster and J. N. Elgin, Asymptotic analysis of the Michelson system, Nonlinearity 16 2149-2162 (2003). 
[23] D. Wilczak, Symmetric heteroclinic connections in the Michelson system: a computer assisted proof, SIAM J. Appl. Dyn. Syst. 4 (2005) 489-514 (electronic). 